\title{
On Organizing Principles of Discrete Differential Geometry. Geometry of spheres
}

\author{
Alexander I. Bobenko * Yuri B. Suris ${ }^{\dagger}$
}

February 2, 2008

\begin{abstract}
Discrete differential geometry aims to develop discrete equivalents of the geometric notions and methods of classical differential geometry. In this survey we discuss the following two fundamental Discretization Principles: the transformation group principle (smooth geometric objects and their discretizations are invariant with respect to the same transformation group) and the consistency principle (discretizations of smooth parametrized geometries can be extended to multidimensional consistent nets). The main concrete geometric problem discussed in this survey is a discretization of curvature line parametrized surfaces in Lie geometry. By systematically applying the Discretization Principles we find a discretization of curvature line parametrization which unifies the circular and conical nets.
\end{abstract}

${ }^{*}$ Institut für Mathematik, Technische Universität Berlin, Str. des 17. Juni 136, 10623 Berlin, Germany. E-mail: bobenko@math.tu-berlin.de

${ }^{\dagger}$ Zentrum Mathematik, Technische Universität München, Boltzmannstr. 3, 85747 Garching bei München, Germany. E-mail: suris@ma.tum.de

Research for this article was supported by the DFG Research Unit 565 "Polyhedral Surfaces" and the DFG Research Center MATheON "Mathematics for key technologies" in Berlin. 


\section{Introduction}

The new field of discrete differential geometry is presently emerging on the border between differential and discrete geometry. Whereas classical differential geometry investigates smooth geometric shapes, discrete differential geometry studies geometric shapes with finite numbers of elements and aims to develop discrete equivalents of the geometric notions and methods of classical differential geometry. The latter appears then as a limit of refinements of the discretization. Current interest in this field derives not only from its importance in pure mathematics but also from its relevance for computer graphics. An important example one should keep in mind here are polyhedral surfaces approximating smooth surfaces.

One may suggest many different reasonable discretizations with the same smooth limit. Which one is the best? From the theoretical point of view the best discretization is the one which preserves all fundamental properties of the smooth theory. Often such a discretization clarifies the structures of the smooth theory and possesses important connections to other fields of mathematics (projective geometry, integrable systems, algebraic geometry, complex analysis etc.). On the other hand, for applications the crucial point is the approximation: the best discretization is supposed to possess distinguished convergence properties and should represent a smooth shape by a discrete shape with just few elements. Although these theoretical and applied criteria for the best discretization are completely different, in many cases natural "theoretical" discretizations turn out to possess remarkable approximation properties and are very useful for applications BSch, LPWYW.

This interaction of the discrete and smooth versions of the theory led to important results in the surface theory as well as in the geometry of polyhedra. Classical achievements of discrete differential geometry are the fundamental results of Alexandrov and Pogorelov on metric geometry of polyhedra and convex surfaces: Alexandrov's theorem [Al] states that any abstract convex polyhedral metric is uniquely realized by a convex polyhedron in Euclidean 3-space. Pogorelov proved Pog the corresponding existence and uniqueness result for abstract convex metrics by approximating smooth surfaces by polyhedra.

Simplicial surfaces, i.e., discrete surfaces made from triangles, are basic in computer graphics. This class of discrete surfaces, however, is too unstructured for analytical investigation. An important tool in the theory of smooth surfaces is the introduction of (special) parametrizations of a surface. Natural analogues of parametrized surfaces are quadrilateral sur- 
faces, i.e. discrete surfaces made from (not necessarily planar) quadrilaterals. The strips of quadrilaterals obtained by gluing quadrilaterals along opposite edges are analogs of coordinate lines. Probably the first nontrivial example of quadrilateral surfaces studied this way are discrete surfaces with constant negative Gaussian curvature introduced by Sauer and Wunderlich S1, Wu. Currently discrete parametrized surfaces are becoming more important in computer graphics. They lead to meshes that better represent the shape of the surface and look regular [ACSDLD, DKG, [MK, [LPWYW].

It is well known that differential equations describing interesting special classes of surfaces and parametrizations are integrable (in the sense of the theory of integrable systems), and, conversely, many of interesting integrable systems admit a differential-geometric interpretation. A progress in understanding of the unifying fundamental structure the classical differential geometers were looking for, and simultaneously in understanding of the very nature of integrability, came from the efforts to discretize these theories. It turns out that many sophisticated properties of differential-geometric objects find their simple explanation within the discrete differential geometry. The early period of this development is documented in the work of Sauer [S2]. The modern period began with the work by Bobenko and Pinkall [BP1, BP2] and by Doliwa and Santini DS, CDS. A closely related development of the spectral theory of difference operators on graphs was initiated by Novikov with collaborators [ND, N1, [N2, see also [DN] for a further development of a discrete complex analysis on simplicial manifolds.

Discrete surfaces in Euclidean 3-space is the basic example considered in this survey. This case has all essential features of the theory in all generality, generalizations for higher dimensions are straightforward. On the other hand, our geometric three-dimensional intuition helps to understand their properties.

Discrete differential geometry related to integrable systems deals with multidimensional discrete nets, i.e., maps from the regular cubic lattice $\mathbb{Z}^{m}$ into $\mathbb{R}^{N}$ specified by certain geometric properties (as mentioned above, we will be most interested in the case $N=3$ in this survey). In this setting discrete surfaces appear as two dimensional layers of multidimensional discrete nets, and their transformations correspond to shifts in the transversal lattice directions. A characteristic feature of the theory is that all lattice directions are on equal footing with respect to the defining geometric properties. Discrete surfaces and their transformations become indistinguishable. We associate such a situation with the multidimensional consistency, and this is one of our fundamental discretization principles. The multidimensional consistency, and therefore the existence and construction of multidimensional 
nets, relies just on certain incidence theorems of elementary geometry.

Conceptually one can think of passing to a continuum limit by refining mesh size in some of the lattice directions. In these directions the net converges to smooth surfaces whereas those directions that remain discrete correspond to transformations of the surfaces (see Fig. 1). The smooth
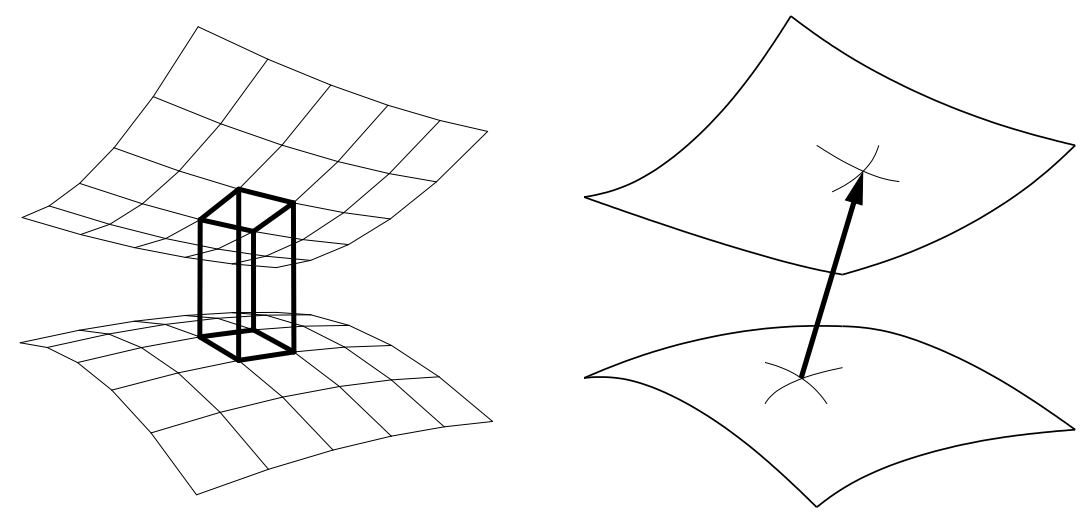

Figure 1: From the discrete master theory to the classical theory: surfaces and their transformations appear by refining two of three net directions.

theory comes as a corollary of a more fundamental discrete master theory. The true roots of the classical surface theory are found, quite unexpectedly, in various incidence theorems of elementary geometry. This phenomenon, which has been shown for many classes of surfaces and coordinate systems BP2, BS2, currently is getting accepted as one of the fundamental features of classical integrable differential geometry.

Note that finding simple discrete explanations for complicated differential geometric theories is not the only outcome of this development. Having identified the roots of the integrable differential geometry in the multidimensional consistency of discrete nets, we are led to a new (geometric) understanding of the integrability itself [BS1, ABS, BS2].

The simplest and at the same time the basic example of consistent multidimensional nets are multidimensional Q-nets [DS], or discrete conjugate nets S2], which are characterized by planarity of all quadrilaterals. The planarity property is preserved by projective transformations and thus Q-nets are subject of projective geometry (like conjugate nets, which are smooth counterparts of Q-nets).

Here we come to the next basic discretization principle. According to F. Klein's Erlangen program, geometries are classified by their transforma- 
tion groups. Classical examples are projective, affine, Euclidean, spherical, hyperbolic geometry, and sphere geometries of Lie, Möebius, and Laguerre. We postulate that the transformation group as the most fundamental feature should be preserved by a discretization. This can be seen as a sort of discrete Erlangen program.

Thus we come to the following fundamental

\section{Discretization Principles:}

- Transformation group principle: smooth geometric objects and their discretizations belong to the same geometry, i.e. are invariant with respect to the same transformation group.

- Consistency principle: discretizations of smooth parametrized geometries can be extended to multidimensional consistent nets.

Let us explain why such different imperatives as the transformation group principle and the consistency principle can be simultaneously imposed for discretization of classical geometries. The transformation groups of various geometries, including those of Lie, Möbius and Laguerre, are subgroups of the projective transformation group. Classically, such a subgroup is described as consisting of projective transformations which preserve some distinguished quadric called absolute. A remarkable result by Doliwa D1] is that multidimensional Q-nets can be restricted to an arbitrary quadric. This is the reason why the Discretization Principles work for the classical geometries.

In this survey we deal with three classical geometries described in terms of spheres: Möbius, Laguerre and Lie geometries. They have been developed by classics, the most elaborated presentation of these geometries can be found in Blaschke's book B1].

Möbius geometry is the most popular one of these three geometries. It describes properties invariant with respect to Möbius transformations which are compositions of reflections in spheres. For $N \geq 3$, Möbius transformations of $\mathbb{R}^{N}$ coincide with conformal transformations. Möbius geometry does not distinguish between spheres and planes (planes are regarded as spheres through the infinitely remote point $\infty$, which compactifies $\mathbb{R}^{N}$ to the $N$ sphere $\mathbb{S}^{N}$ ). On the other hand, points are considered as objects different from spheres. Surfaces are described through their points. Classical examples of Möbius-geometric properties of surfaces are conformal parametrization and the Willmore functional Wi]. Recent progress in this field is to a large extent due to interrelations with the theory of integrable systems [FLPP, T]. 
Laguerre geometry does not distinguish points and spheres (points are treated as spheres of zero radius). On the other hand, planes are distinguished. Surfaces are described through their tangent planes. A particular Laguerre transformation of a surface is a shift of all tangent planes in the normal direction at a constant distance. This transformation is called the normal shift.

Lie geometry is a natural unification of Möbius and Laguerre geometries: points, planes and spheres are treated on an equal footing. The transformation group is generated by Möbius transformations and the normal shift transformations. Surfaces are described through their contact elements. A contact element can be understood as a surface point together with the corresponding tangent plane. The one-parameter family of spheres through a point with a common tangent plane gives a Lie-geometric description of a contact element. The point of the surface and the tangent plane at this point are just two elements of this family.

Integrability aspects of the surface theory in Lie geometry have been studied by Ferapontov [F1, F2, Musso and Nicolodi [MN], and Burstall and Hertrich-Jeromin BHJ1, BHJ2].

The main concrete geometric problem discussed in this survey is a discretization of curvature line parametrized surfaces. Curvature lines are integral curves of the principal directions. Any surface away from its umbilic points can be parametrized by curvature lines. Curvature line parametrization is attracting the attention of mathematicians and physicists for two centuries. The classical results in this field can be found in the books by Darboux Da1, Da2 and Bianchi Bi]. In particular a classical result of Dupin [D] claims that the coordinate surfaces of triply orthogonal coordinate systems intersect along their common curvature lines. Ribaucour has discovered a transformation of surfaces preserving the curvature line parametrization (see [E2]). A surface and its Ribaucour transform envelope a special sphere congruence. Bianchi has shown Bi2] that Ribaucour transformations are permutable: given two Ribaucour transforms of a surface there exists a one-parameter family of their common Ribaucour transforms.

Recently curvature line parametrizations and orthogonal systems came back into the focus of interest in mathematical physics as an example of an integrable system. Zakharov [Z] has constructed a variety of explicit solutions with the help of the dressing method. Algebro-geometric orthogonal coordinate systems were constructed by Krichever $[\mathrm{K}]$. The recent interest to this problem is in particular motivated by applications to the theory of the associativity equations developed by Dubrovin [Du]. Remarkable geometric properties make curvature line parametrizations especially useful for 
visualization of surfaces in computer graphics [ACSDLD, LPWYW].

The question of proper discretization of the curvature line parametrized surfaces and orthogonal systems became recently a subject of intensive study. Circular nets, which are Q-nets with circular quadrilaterals, as discrete analogs of curvature line parametrized surfaces were mentioned by Nutbourne and Martin $[\mathrm{NM}$. Special circular nets as discrete isothermic surfaces were investigated in $\mathrm{BP} 2$. The circular discretization of triplyorthogonal coordinate systems was first suggested by one of the authors in [B]. Doliwa and Santini [DS] made the next crucial step in the development of the theory. They considered discrete orthogonal systems as a reduction of discrete conjugated systems [CDS], generalized them to arbitrary dimension and proved their multidimensional consistency based on the classical Miquel theorem [Be].

Matthes and the authors of this survey have proven [BMS] that circular nets approximate smooth curvature line parametrized surfaces and orthogonal systems with all derivatives. Numerical experiments show that circular nets have the desired geometrical properties already at the coarse level and not only in the refinement limit as it approaches a smooth curvature line parameterized surface. This is important for applications in computer graphics [LPWYW].

A convenient analytic description of circular nets has been given by Konopelchenko and Schief [KS]. Analytic methods of the soliton theory have been applied to circular nets by Doliwa, Manakov and Santini DMS] ( $\bar{\partial}$-method) and by Akhmetshin, Volvovskii and Krichever AKV (algebrogeometric solutions). Bobenko and Hertrich-Jeromin [BHJ] have given a Clifford algebra description of circular nets.

Circular nets are preserved by Möbius transformations, and thus should be treated as the discretization of curvature line parametrizations in Möbius geometry. A remarkable recent development by Liu, Pottmann, Wallner, Yang, and Wang [LPWYW] is the introduction of conical nets, which should be treated as the discretization of curvature line parametrizations in Laguerre geometry. These are special Q-nets characterized by the property that four quadrilaterals meeting at a vertex are tangent to a common cone of revolution. Equivalently, conical nets can be characterized as Q-nets with circular Gauss maps, i.e., the unit normals to the quadrilaterals comprise a circular net in the unit sphere $S^{2}$. Circular Gauss maps defined at vertices of a given circular net were previously introduced by Schief Sch2, however without relation to conical nets. Conical nets, like circular ones, satisfy the second discretization principle (consistency).

In the present survey, we find a discretization of curvature line parametriza- 
tion which unifies the circular and the conical nets by systematically applying the Discretization Principles.

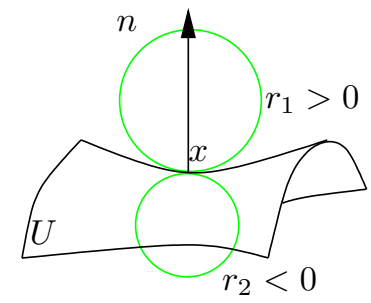

Figure 2: Principal directions through touching spheres.

It is well known that curvature lines are subject of Lie geometry, i.e., are invariant with respect to Möbius transformations and normal shifts. To see this, consider an infinitesimal neighborhood $U$ of a point $x$ of an oriented smooth surface in $\mathbb{R}^{3}$, and the pencil of spheres $S(r)$ of the signed radii $r$, touching the surface at $x$, see Fig. 2. The signed radius $r$ is assumed positive if $S(r)$ lies on the same side of the surface as the normal $n$, and negative otherwise; $S(\infty)$ is the tangent plane. For small $r_{0}>0$ the spheres $S\left(r_{0}\right)$ and $S\left(-r_{0}\right)$ intersect $U$ in $x$ only. The set of the touching spheres with this property (intersecting $U$ in $x$ only) has two connected components: $M_{+}$ containing $S\left(r_{0}\right)$ and $M_{-}$containing $S\left(-r_{0}\right)$ for small $r_{0}>0$. The boundary values

$$
r_{1}=\sup \left\{r: S(r) \in M_{+}\right\}, \quad r_{2}=\inf \left\{r: S(r) \in M_{-}\right\}
$$

are the principal curvatures of the surface in $x$. The directions in which $S\left(r_{1}\right)$ and $S\left(r_{2}\right)$ touch $U$ are the principal directions.

Clearly, all ingredients of this description are Möbius-invariant. Under a normal shift by the distance $d$ the centers of the principal curvature spheres are preserved and their radii are shifted by $d$. This implies that the principal directions and thus the curvature lines are preserved under normal shifts, as well.

A Lie-geometric nature of the curvature line parametrization yields that it has a Lie-invariant description. Such a description can be found in Blaschke's book B1]. A surface in Lie geometry, as already said, is considered as consisting of contact elements. Two infinitesimally close contact elements (sphere pencils) belong to the same curvature line, if and only if they have a sphere in common, which is the principal curvature sphere. 
By a literal discretization of this Blaschke's Lie-geometric description of smooth curvature line parametrized surfaces, we define a discrete principal contact element net as a map $\mathbb{Z}^{2} \rightarrow$ contact elements of surfaces in $\mathbb{R}^{3}$ \} such that any two neighboring contact elements have a sphere in common.

In the projective model of Lie geometry spheres in $\mathbb{R}^{3}$ (including points and planes) are represented by elements of the so called Lie quadric $\mathbb{L} \subset \mathbb{R} \mathbb{P}^{5}$, contact elements are represented by isotropic lines, i.e., lines in $\mathbb{L}$, surfaces are represented by congruences of isotropic lines. In the curvature line parametrization, the parametric families of isotropic lines comprise developable surfaces in $\mathbb{L}$.

Accordingly, a discrete principal contact element net in the projective model of Lie geometry is a discrete congruence of isotropic lines

$$
\ell: \mathbb{Z}^{2} \rightarrow\{\text { isotropic lines in } \mathbb{L}\}
$$

such that any two neighboring lines intersect. Intersection points of neighboring lines are, as in the smooth case, the principal curvature spheres. They are associated with the edges of $\mathbb{Z}^{2}$. Four principal curvature spheres associated to the edges with a common vertex belong to the same contact element, i.e., have a common touching point.

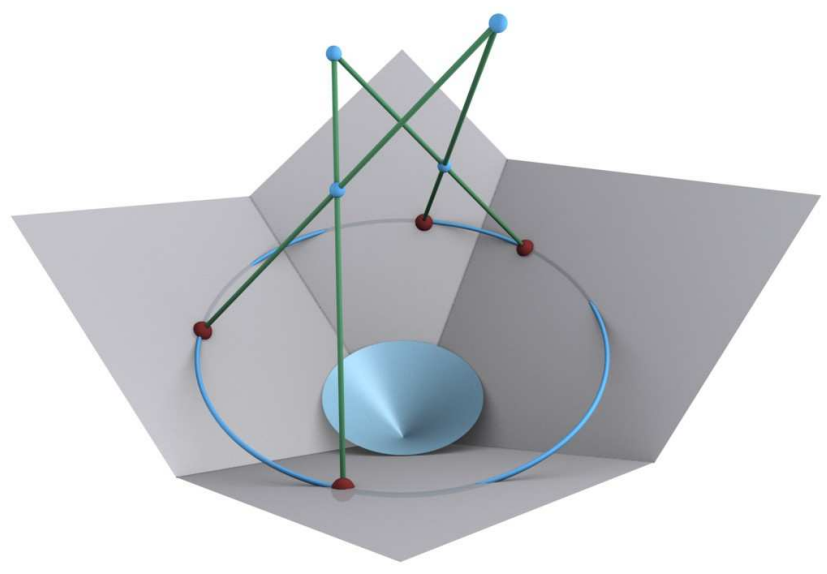

Figure 3: Geometry of principal contact element nets. Four neighboring contact elements are represented by points and (tangent) planes. The points are concircular, the planes are tangent to a cone of revolution. Neighboring normal lines intersect at the centers of principal curvature spheres. 
In projective geometry, discrete line congruences have been introduced by Doliwa, Mañas and Santini [DMS]. Discrete line congruences are closely related to Q-nets, and, like the latter, are multidimensionally consistent. It follows from our results that they can be restricted to the Lie quadric (actually, to any ruled quadric). Thus, principal contact element nets satisfy the second discretization principle. In particular, this yields discrete Ribaucour transformations between principal contact element nets.

The Lie-geometric notion of discrete principal contact element nets unifies the Möbius-geometric one (circular nets) and the Laguerre-geometric one (conical nets). Indeed, any contact element $\ell$ contains a point $x$ and a plane $P$. It turns out that for a surface

$$
\ell: \mathbb{Z}^{2} \rightarrow\{\text { isotropic lines in } \mathbb{L}\}=\left\{\text { contact elements in } \mathbb{R}^{3}\right\},
$$

the points comprise a circular net

$$
x: \mathbb{Z}^{2} \rightarrow \mathbb{R}^{3},
$$

whereas the planes comprise a conical net

$$
P: \mathbb{Z}^{2} \rightarrow\left\{\text { planes in } \mathbb{R}^{3}\right\}
$$

The corresponding geometry is depicted on Fig. 3. Schematically, this Liegeometric merging of the Möbius- and Laguerre-geometric notions is presented on Fig. 4.

This survey is organized as follows. In Sect. 2 we start with a review of the basic multidimensionally consistent systems - the Q-nets and discrete line congruences. The basic notions of Lie, Möbius and Laguerre geometries are briefly presented in Sect. 3. Sect. 4] contains main new results on discrete curvature line parametrized surfaces: Lie-geometric Definitions 18, 19, and Theorem 32 which describes interrelations of discrete curvature line nets in Lie, Möbius and Laguerre geometries. Geometric characterization of Ribaucour transformations and discrete R-congruences of spheres as quadrilateral nets in the Lie quadric is given in Sect. 5 .

Let us note that, due to the classical Lie's sphere-line correspondence, the Lie-geometric theory presented in this survey can be transferred to the context of projective line geometry in three-space: the Lie quadric is replaced by the Plücker quadric, the curvature lines and R-congruences of spheres correspond to the asymptotic lines and the $\mathrm{W}$-congruences of lines, respectively. The projective theory of discrete asymptotic nets has been developed by Doliwa [D2]. 


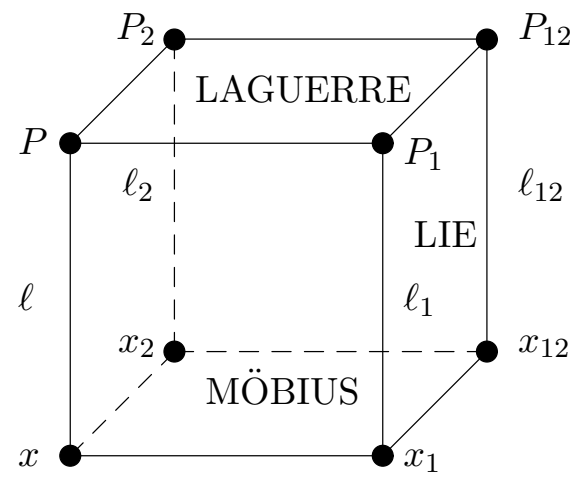

Figure 4: Geometry of principal contact element nets. Four neighboring contact elements produce a hexahedron with vertices in the Lie quadric $\mathbb{L}$ and with planar faces. The bottom quadrilateral is the intersection of the three-dimensional space $V=\operatorname{span}\left(\ell, \ell_{1}, \ell_{2}, \ell_{12}\right)$ with the 4 -space in $\mathbb{R} \mathbb{P}^{5}$ representing points in $\mathbb{R}^{3}$. The top quadrilateral is the intersection of $V$ with the 4 -space in $\mathbb{R P}^{5}$ representing planes in $\mathbb{R}^{3}$. Each side quadrilateral lies in the plane of two intersecting lines $\ell \subset \mathbb{L}$.

Our research in discrete differential Lie geometry has been stimulated by the recent introduction of conical nets by Liu, Pottmann, Wallner, Yang, and Wang [LPWY]. The advent of the second (after circular nets) discretization of curvature line parametrizations posed a question about the relation between the different discretizations. Independently, a relation between circular and conical nets has been found by Pottmann $[\mathrm{P}$. We are grateful to H. Pottmann and J. Wallner for numerous communications on conical nets and for providing us with their unpublished results. We thank also U. Pinkall for useful discussions.

\section{Consistency as a discretization principle}

\subsection{Q-nets}

We use the following standard notation: for a function $f$ on $\mathbb{Z}^{m}$ we write

$$
\tau_{i} f(u)=f\left(u+e_{i}\right)
$$

where $e_{i}$ is the unit vector of the $i$-th coordinate direction, $1 \leq i \leq m$. We use also the shortcut notations $f_{i}$ for $\tau_{i} f, f_{i j}$ for $\tau_{i} \tau_{j} f$, etc. 
The most general of the known discrete 3D systems possessing the property of $4 \mathrm{D}$ consistency are nets consisting of planar quadrilaterals, or Qnets. Two-dimensional Q-nets were introduced by Sauer [S2, the multidimensional generalization has been given by Doliwa and Santini [DS]. Our presentation in this section follows the latter paper. The fundamental importance of multi-dimensional consistency of discrete systems as their integrability has been put forward by the authors [BS1, ABS, BS2].

Definition 1 (Q-net) $A$ map $f: \mathbb{Z}^{m} \rightarrow \mathbb{R P}^{N}$ is called an m-dimensional Q-net (quadrilateral net, or discrete conjugate net) in $\mathbb{R P}^{N}(N \geq 3)$, if all its elementary quadrilaterals $\left(f, f_{i}, f_{i j}, f_{j}\right.$ ) (at any $u \in \mathbb{Z}^{m}$ and for all pairs $1 \leq i \neq j \leq m$ ) are planar.

Thus, for any elementary quadrilateral, any representatives $\tilde{f}, \tilde{f}_{i}, \tilde{f}_{j}, \tilde{f}_{i j}$ of its vertices in the space $\mathbb{R}^{N+1}$ of homogeneous coordinates satisfy an equation of the type

$$
\tilde{f}_{i j}=c_{i j} \tilde{f}_{j}+c_{j i} \tilde{f}_{i}+\rho_{i j} \tilde{f} .
$$

Representatives in any hyperplane of $\mathbb{R}^{N+1}$, for instance, in the affine part $\mathbb{R}^{N}$ of $\mathbb{R} \mathbb{P}^{N}=\mathbb{P}\left(\mathbb{R}^{N+1}\right)$, satisfy such an equation with $1=c_{i j}+c_{j i}+\rho_{i j}$, that is,

$$
\tilde{f}_{i j}-\tilde{f}=c_{i j}\left(\tilde{f}_{j}-\tilde{f}\right)+c_{j i}\left(\tilde{f}_{i}-\tilde{f}\right) .
$$

Given three points $f, f_{1}, f_{2}$ in $\mathbb{R} \mathbb{P}^{N}$, one can take any point of the plane through these three points as the fourth vertex $f_{12}$ of an elementary quadrilateral $\left(f, f_{1}, f_{12}, f_{2}\right)$ of a Q-net. Correspondingly, given any two discrete curves $f: \mathbb{Z} \times\{0\} \rightarrow \mathbb{R P}^{N}$ and $f:\{0\} \times \mathbb{Z} \rightarrow \mathbb{R P}^{N}$ with a common point $f(0,0)$, one can construct infinitely many Q-surfaces $f: \mathbb{Z}^{2} \rightarrow \mathbb{R} \mathbb{P}^{N}$ with these curves as coordinate ones: the construction goes inductively, on each step one has a freedom of choosing a point in a plane (two real parameters).

On the other hand, constructing elementary hexahedra of Q-nets corresponding to elementary $3 \mathrm{D}$ cubes of the lattice $\mathbb{Z}^{m}$ admits a well-posed initial value problem with a unique solution, therefore one says that Q-nets are described by a discrete $3 D$ system:

Theorem 2 (Elementary hexahedron of a Q-net) Given seven points $f, f_{i}$ and $f_{i j}(1 \leq i<j \leq 3)$ in $\mathbb{R P}^{N}$, such that each of the three quadrilaterals $\left(f, f_{i}, f_{i j}, f_{j}\right)$ is planar (i.e., $f_{i j}$ lies in the plane $\Pi_{i j}$ through $\left.f, f_{i}, f_{j}\right)$, define three planes $\tau_{k} \Pi_{i j}$ as those passing through the point triples $f_{k}, f_{i k}$, $f_{j k}$, respectively. Then these three planes intersect generically at one point:

$$
f_{123}=\tau_{1} \Pi_{23} \cap \tau_{2} \Pi_{13} \cap \tau_{3} \Pi_{12} .
$$


Proof. Planarity of the quadrilaterals $\left(f, f_{i}, f_{i j}, f_{j}\right)$ assures that all seven initial points $f, f_{i}$ and $f_{i j}$ belong to the three-dimensional space $\Pi_{123}$ through the four points $f, f_{1}, f_{2}, f_{3}$. Hence, the planes $\tau_{k} \Pi_{i j}$ lie in this threedimensional space, and therefore generically they intersect at exactly one point.

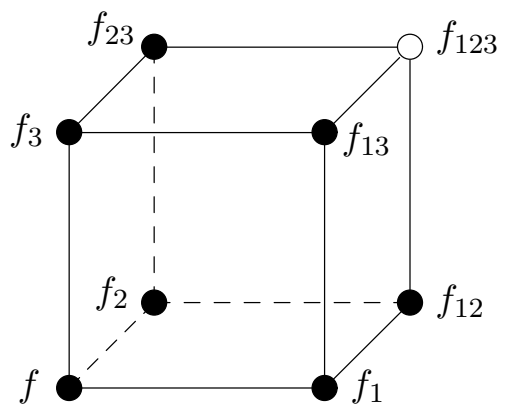

Figure 5: 3D system on an elementary cube

The elementary construction step from Theorem 2 is symbolically represented on Fig. 5, which is the picture we have in mind when thinking and speaking about discrete three-dimensional systems with dependent variables (fields) attached to the vertices of a regular cubic lattice.

As follows from Theorem 2] a three-dimensional Q-net $f: \mathbb{Z}^{3} \rightarrow \mathbb{R} \mathbb{P}^{N}$ is completely determined by its three coordinate surfaces

$$
f: \mathbb{Z}^{2} \times\{0\} \rightarrow \mathbb{R P}^{N}, \quad f: \mathbb{Z} \times\{0\} \times \mathbb{Z} \rightarrow \mathbb{R P}^{N}, \quad f:\{0\} \times \mathbb{Z}^{2} \rightarrow \mathbb{R P}^{N} .
$$

Turning to an elementary cube of the dimension $m \geq 4$, we see that one can prescribe all points $f, f_{i}$ and $f_{i j}$ for all $1 \leq i<j \leq m$. Indeed, these data are clearly independent, and one can construct all other vertices of an elementary cube starting from these data, provided one does not encounter contradictions. To see the possible source of contradictions, consider in detail first the case of $m=4$. From $f, f_{i}$ and $f_{i j}(1 \leq i<j \leq 4)$ one determines all $f_{i j k}$ uniquely. After that, one has, in principle, four different ways to determine $f_{1234}$, from four $3 \mathrm{D}$ cubic faces adjacent to this point; see Fig. 6] Absence of contradictions means that these four values for $f_{1234}$ automatically coincide. We call this property the $4 \mathrm{D}$ consistency.

Definition 3 ( $4 \mathrm{D}$ consistency) A $3 D$ system is called $4 D$ consistent, if it can be imposed on all three-dimensional faces of an elementary cube of $\mathbb{Z}^{4}$. 


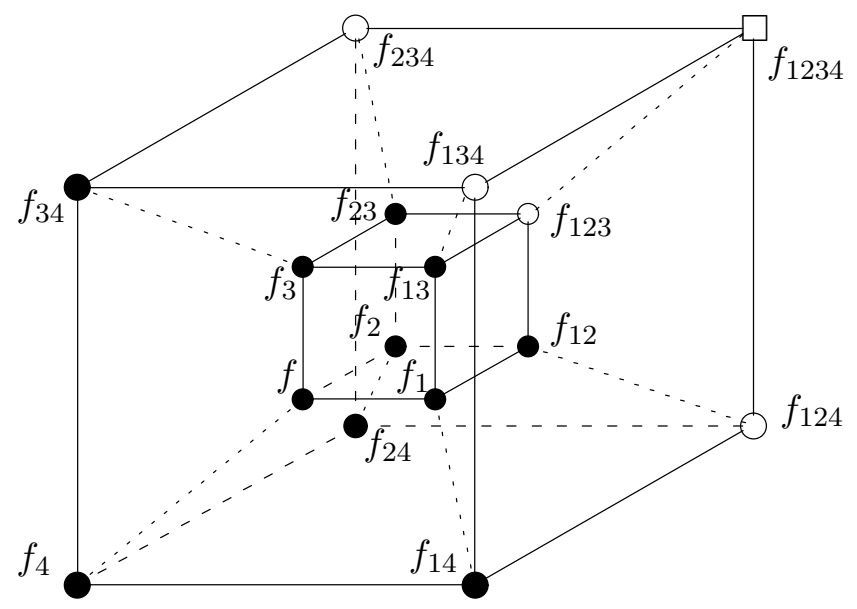

Figure 6: 4D consistency of 3D systems

Remarkably, construction of Q-nets based on the planarity of all elementary quadrilaterals enjoys this property.

Theorem 4 (Q-nets are $4 \mathrm{D}$ consistent) The $3 D$ system governing $Q$ nets is $4 D$-consistent.

Proof. In the construction above, the four values in question are

$$
f_{1234}=\tau_{1} \tau_{2} \Pi_{34} \cap \tau_{1} \tau_{3} \Pi_{24} \cap \tau_{1} \tau_{4} \Pi_{23},
$$

and three other ones obtained by cyclic shifts of indices. Thus, we have to prove that the six planes $\tau_{i} \tau_{j} \Pi_{k \ell}$ intersect in one point.

First, assume that the ambient space $\mathbb{R P}^{N}$ has dimension $N \geq 4$. Then, in general position, the space $\Pi_{1234}$ through the five points $f, f_{i}(1 \leq i \leq 4)$ is four-dimensional. It is easy to understand that the plane $\tau_{i} \tau_{j} \Pi_{k \ell}$ is the intersection of two three-dimensional subspaces $\tau_{i} \Pi_{j k \ell}$ and $\tau_{j} \Pi_{i k \ell}$. Indeed, the subspace $\tau_{i} \Pi_{j k \ell}$ through the four points $f_{i}, f_{i j}, f_{i k}, f_{i \ell}$ contains also $f_{i j k}$, $f_{i j \ell}$, and $f_{j k \ell}$. Therefore, both $\tau_{i} \Pi_{j k \ell}$ and $\tau_{j} \Pi_{i k \ell}$ contain three points $f_{i j}$, $f_{i j k}$ and $f_{i j \ell}$, which determine the plane $\tau_{i} \tau_{j} \Pi_{k \ell}$. Now the intersection in question can be alternatively described as the intersection of the four threedimensional subspaces $\tau_{1} \Pi_{234}, \tau_{2} \Pi_{134}, \tau_{3} \Pi_{124}$ and $\tau_{4} \Pi_{123}$ of one and the same four-dimensional space $\Pi_{1234}$. This intersection consists in the generic case of exactly one point.

In the case of $N=3$, we embed the ambient space into $\mathbb{R} \mathbb{P}^{4}$, then slightly perturb the point $f_{4}$ by adding a small component in the fourth 
coordinate direction, then apply the above argument, and after that send the perturbation to zero. This proof works since, as one can easily see, on each step of the construction the perturbation remains regular.

The $m$-dimensional consistency of a $3 \mathrm{D}$ system for $m>4$ is defined analogously to the $m=4$ case. Remarkably and quite generally, the 4dimensional consistency already implies $m$-dimensional consistency for all $m>4$.

Theorem 5 (4D consistency yields consistency in all higher dimensions) Any $4 D$ consistent discrete $3 D$ system is also $m$-dimensionally consistent for any $m>4$.

Proof goes by induction from the $(m-1)$-dimensional consistency to the $m$ dimensional consistency, but, for the sake of notational simplicity, we present the details for the case $m=5$ only, the general case being absolutely similar.

Initial data for a $3 \mathrm{D}$ system on the $5 \mathrm{D}$ cube $\mathcal{C}_{12345}$ with the fields on vertices consist of the fields $f, f_{i}$ and $f_{i j}$ for all $1 \leq i<j \leq 5$. From these data one first gets ten fields $f_{i j k}$ for $1 \leq i<j<k \leq 5$, and then five fields $f_{i j k \ell}$ for $1 \leq i<j<k<\ell \leq 5$ (the fact that the latter are well defined is nothing but the assumed $4 \mathrm{D}$ consistency for the $4 \mathrm{D}$ cubes $\left.\mathrm{C}_{i j k l}\right)$. Now, one has ten possibly different values for $f_{12345}$, coming from ten $3 \mathrm{D}$ cubes $\tau_{i} \tau_{j} \mathcal{C}_{k \ell m}$. To prove that these ten values coincide, consider five $4 \mathrm{D}$ cubes $\tau_{i} \mathrm{e}_{j k \ell m}$. For instance, for the $4 \mathrm{D}$ cube $\tau_{1} \mathrm{e}_{2345}$ the assumed consistency assures that the four values for $f_{12345}$ coming from four $3 \mathrm{D}$ cubes

$$
\tau_{1} \tau_{2} \mathfrak{C}_{345}, \quad \tau_{1} \tau_{3} \mathfrak{C}_{245}, \quad \tau_{1} \tau_{4} \mathfrak{C}_{235}, \quad \tau_{1} \tau_{5} \mathfrak{C}_{234}
$$

are all the same. Similarly, for the $4 \mathrm{D}$ cube $\tau_{2} \mathrm{C}_{1345}$ the $4 \mathrm{D}$ consistency leads to the conclusion that the four values for $f_{12345}$ coming from

$$
\tau_{1} \tau_{2} \mathcal{C}_{345}, \quad \tau_{2} \tau_{3} \mathfrak{C}_{145}, \quad \tau_{2} \tau_{4} \mathfrak{C}_{135}, \quad \tau_{2} \tau_{5} \mathfrak{C}_{134}
$$

coincide. Note that the $3 \mathrm{D}$ cube $\tau_{1} \tau_{2} \mathrm{C}_{345}$, the intersection of $\tau_{1} \mathrm{C}_{2345}$ and $\tau_{2} \mathrm{e}_{1345}$, is present in both lists, so that we now have seven coinciding values for $f_{12345}$. Adding similar conclusions for other $4 \mathrm{D}$ cubes $\tau_{i} \mathrm{e}_{j k \ell m}$, we arrive at the desired result.

Theorems 4 5yield that Q-nets are $m$-dimensionally consistent for any $m \geq 4$. This fact, in turn, yields the existence of transformations of Q-nets with remarkable permutability properties. Referring for details to [DSM, BS2, we mention here only the definition. 
Definition 6 (F-transformation of Q-nets) Two m-dimensional Q-nets $f, f^{+}: \mathbb{Z}^{m} \rightarrow \mathbb{R P}^{N}$ are called F-transforms (fundamental transforms) of one another, if all quadrilaterals $\left(f, f_{i}, f_{i}^{+}, f^{+}\right.$) (at any $u \in \mathbb{Z}^{m}$ and for all $1 \leq i \leq m$ ) are planar, i.e., if the net $F: \mathbb{Z}^{m} \times\{0,1\} \rightarrow \mathbb{R P}^{N}$ defined by $F(u, 0)=f(u)$ and $F(u, 1)=f^{+}(u)$ is a two-layer $(m+1)$-dimensional Q-net.

It follows from Theorem 2 that, given a Q-net $f$, its F-transform $f^{+}$is uniquely defined as soon as its points along the coordinate axes are suitably prescribed.

\section{$2.2 \quad$ Discrete line congruences}

Another important geometrical objects described by a discrete 3D system which is $4 \mathrm{D}$ consistent, are discrete line congruences. Their theory has been developed by Doliwa, Santini and Mañas [DSM], whose presentation we follow in this section.

Let $\mathcal{L}^{N}$ be the space of lines in $\mathbb{R} \mathbb{P}^{N}$; it can be identified with the Grassmannian $\operatorname{Gr}(N+1,2)$ of two-dimensional vector subspaces of $\mathbb{R}^{N+1}$.

Definition 7 (Discrete line congruence) $A$ map $\ell: \mathbb{Z}^{m} \rightarrow \mathcal{L}^{N}$ is called an $m$-dimensional discrete line congruence in $\mathbb{R P}^{N}(N \geq 3)$, if any two neighboring lines $\ell, \ell_{i}$ (at any $u \in \mathbb{Z}^{m}$ and for any $1 \leq i \leq m$ ) intersect (are co-planar).

For instance, lines $\ell=\left(f f^{+}\right)$connecting corresponding points of two Q-nets $f, f^{+}: \mathbb{Z}^{m} \rightarrow \mathbb{R P}^{N}$ in the relation of F-transformation clearly build a discrete line congruence.

A discrete line congruence is called generic, if for any $u \in \mathbb{Z}^{m}$ and for any $1 \leq i \neq j \neq k \neq i \leq m$, the four lines $\ell, \ell_{i}, \ell_{j}$ and $\ell_{k}$ span a fourdimensional space (i.e., a space of a maximal possible dimension). This yields, in particular, that for any $u \in \mathbb{Z}^{m}$ and for any $1 \leq i \neq j \leq m$, the three lines $\ell, \ell_{i}$ and $\ell_{j}$ span a three-dimensional space.

Construction of line congruences is similar to that of Q-nets. Given three lines $\ell, \ell_{1}, \ell_{2}$ of a congruence, one has a two-parameter family of lines admissible as the fourth one $\ell_{12}$ : connect by a line any point of $\ell_{1}$ with any point of $\ell_{2}$. Thus, given any two sequences of lines $\ell: \mathbb{Z} \times\{0\} \rightarrow \mathcal{L}^{N}$ and $\ell:\{0\} \times \mathbb{Z} \rightarrow \mathcal{L}^{N}$ such that any two neighboring lines are co-planar, one can extend them to a two-dimensional line congruence $f: \mathbb{Z}^{2} \rightarrow \mathcal{L}^{N}$ in an infinite number of ways: on each step of the inductive procedure one has a freedom of choosing a line from a two-parameter family. 
The next theorem shows that non-degenerate line congruences are described by a discrete $3 D$ system:

Theorem 8 (Elementary hexahedron of a discrete line congruence) Given seven lines $\ell, \ell_{i}$ and $\ell_{i j}(1 \leq i<j \leq 3)$ in $\mathbb{R P}^{N}$, such that $\ell$ intersects each of $\ell_{i}$, the space $V_{123}$ spanned by $\ell, \ell_{1}, \ell_{2}, \ell_{3}$ has dimension four, and each $\ell_{i}$ intersects both $\ell_{i j}$ and $\ell_{i k}$, there is a unique line $\ell_{123}$ that intersects all three $\ell_{i j}$.

Proof. All seven lines, and therefore also the three-dimensional spaces $\tau_{i} V_{j k}=\operatorname{span}\left(\ell_{i}, \ell_{i j}, \ell_{i k}\right)$ lie in $V_{123}$. A line that intersects all three of $\ell_{i j}$ should lie in the intersection of these three three-dimensional spaces. But a generic intersection of three three-dimensional spaces in $V_{123}$ is a line:

$$
\ell_{123}=\tau_{1} V_{23} \cap \tau_{2} V_{13} \cap \tau_{3} V_{12}
$$

It is now not difficult to realize that this line does, indeed, intersect all three of $\ell_{i j}$. For instance, $\tau_{1} V_{23} \cap \tau_{2} V_{13}=\operatorname{span}\left(\ell_{12}, \ell_{13}\right) \cap \operatorname{span}\left(\ell_{12}, \ell_{23}\right)$ is a plane containing $\ell_{12}$, therefore its intersection with $\tau_{3} V_{12}$ (the line $\ell_{123}$ ) intersects $\ell_{12}$.

A similar argument shows:

Theorem 9 (Discrete line congruences are $4 \mathrm{D}$ consistent) The $3 D$ system governing discrete line congruences is $4 D$-consistent.

Like in the case of Q-nets, this theorem yields the existence of transformations of discrete line congruences with remarkable permutability properties.

Definition 10 (F-transformation of line congruences) Two m-dimensional line congruences $\ell, \ell^{+}: \mathbb{Z}^{m} \rightarrow \mathcal{L}^{N}$ are called $F$-transforms of one another, if the corresponding lines $\ell$ and $\ell^{+}$intersect (at any $u \in \mathbb{Z}^{m}$ ), i.e., if the map $L: \mathbb{Z}^{m} \times\{0,1\} \rightarrow \mathcal{L}^{N}$ defined by $L(u, 0)=\ell(u)$ and $L(u, 1)=\ell^{+}(u)$ is a two-layer $(m+1)$-dimensional line congruence.

Again, it follows from Theorem 2 that, given a line congruence $\ell$, its Ftransform $\ell^{+}$is uniquely defined as soon as its lines along the coordinate axes are suitably prescribed.

According to Definition 7 any two neighboring lines $\ell=\ell(u)$ and $\ell_{i}=$ $\ell\left(u+e_{i}\right)$ of a line congruence intersect at exactly one point $f=\ell \cap \ell_{i} \in \mathbb{R P}^{N}$ which is thus combinatorially associated with the edge $\left(u, u+e_{i}\right)$ of the lattice $\mathbb{Z}^{m}: f=f\left(u, u+e_{i}\right)$. It is, however, sometimes more convenient to 


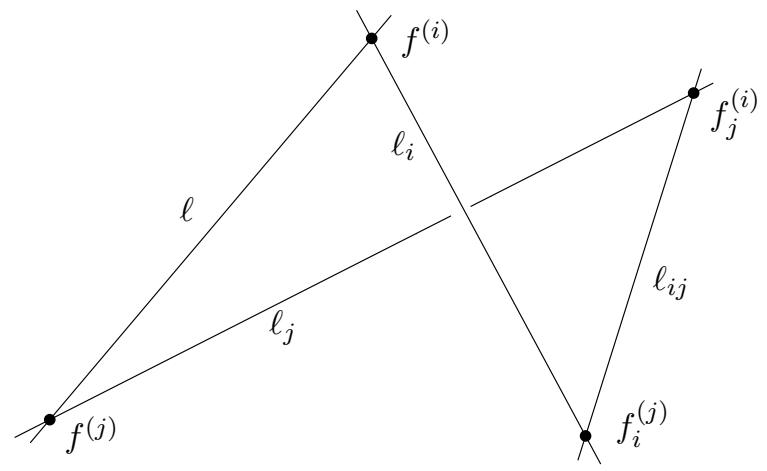

Figure 7: Four lines of a congruence

use the notation $f\left(u, u+e_{i}\right)=f^{(i)}(u)$ for this points, thus associating it to the vertex $u$ of the lattice (and, of course, to the coordinate direction $i$ ). See Fig. 7.

Definition 11 (Focal net) For a discrete line congruence $\ell: \mathbb{Z}^{m} \rightarrow \mathcal{L}^{N}$, the map $f^{(i)}: \mathbb{Z}^{m} \rightarrow \mathbb{R P}^{N}$ defined by $f^{(i)}(u)=\ell(u) \cap \ell\left(u+e_{i}\right)$ is called its $i$-th focal net.

Theorem 12 For a non-degenerate discrete line congruence $\ell: \mathbb{Z}^{m} \rightarrow \mathcal{L}^{N}$, all its focal nets $f^{(k)}: \mathbb{Z}^{m} \rightarrow \mathbb{R P}^{N}, 1 \leq k \leq m$, are Q-nets.

Proof consists of two steps.

- First, one shows that for the $k$-th focal net $f^{(k)}$, all elementary quadrilaterals $\left(f^{(k)}, f_{i}^{(k)}, f_{i k}^{(k)}, f_{k}^{(k)}\right)$ are planar. This is true for any line congruence. Indeed, both points $f^{(k)}$ and $f_{k}^{(k)}$ lie on the line $\ell_{k}$, while both points $f_{i}^{(k)}$ and $f_{i k}^{(k)}$ lie on the line $\ell_{i k}$. Therefore, all four points lie in the plane spanned by these two lines $\ell_{k}$ and $\ell_{i k}$ which intersect by definition of a line congruence.

- Second, one shows that for the $k$-th focal net $f^{(k)}$, all elementary quadrilaterals $\left(f^{(k)}, f_{i}^{(k)}, f_{i j}^{(k)}, f_{j}^{(k)}\right)$, with both $i \neq j$ different from $k$, are planar. Here, one uses essentially the assumption that the line congruence $\ell$ is generic. All four points in question lie in each of the three-dimensional spaces

$$
V_{i j}=\operatorname{span}\left(\ell, \ell_{i}, \ell_{j}, \ell_{i j}\right) \quad \text { and } \tau_{k} V_{i j}=\operatorname{span}\left(\ell_{k}, \ell_{i k}, \ell_{j k}, \ell_{i j k}\right)
$$


(see Fig. 8). Both 3-spaces lie in the four-dimensional space $V_{i j k}=\operatorname{span}\left(\ell, \ell_{i}, \ell_{j}, \ell_{k}\right)$, so that generically their intersection is a plane.

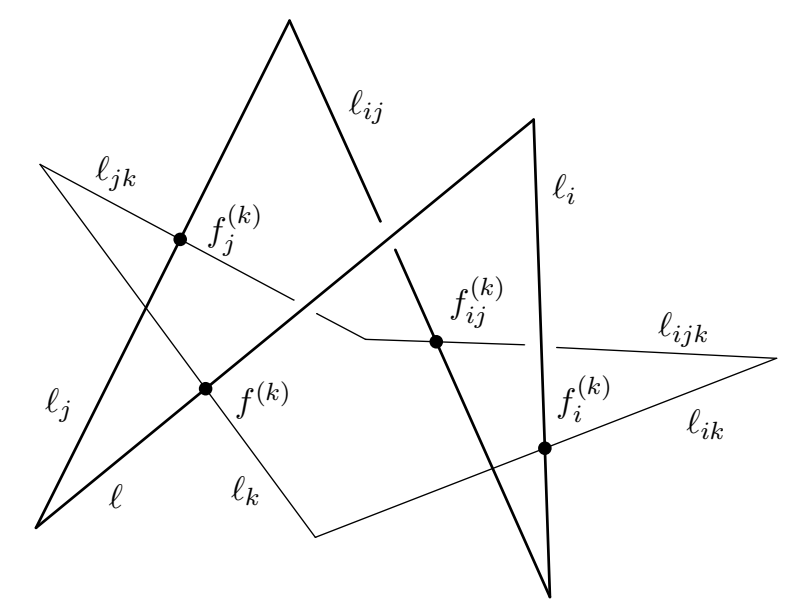

Figure 8: Elementary $(i j)$ quadrilateral of the $k$-th focal net

Corollary 13 (Focal net of F-transformation of a line congruence) Given two generic line congruences $\ell, \ell^{+}: \mathbb{Z}^{m} \rightarrow \mathcal{L}^{N}$ in the relation of $F$ transformation, the intersection points $f=\ell \cap \ell^{+}$form a $Q$-net $f: \mathbb{Z}^{m} \rightarrow$ $\mathbb{R P}^{N}$.

\subsection{Q-nets in quadrics}

We consider an important admissible reduction of Q-nets: they can be consistently restricted to an arbitrary quadric in $\mathbb{R P}^{N}$. In the smooth differential geometry, i.e., for conjugate nets, this is due to Darboux Da1]. In the discrete differential geometry this result has been found by Doliwa [D1].

A deep reason for this result is the following fundamental fact well known in classical projective geometry (see, e.g., [B2]):

Theorem 14 (Associated point) For any seven points of $\mathbb{C P}^{3}$ in general position, there exists the eighth point (called the associated one), which belongs to any quadric through the original seven points. 
Proof is based on the following computations. The equation $Q=0$ of a quadric in $\mathbb{C P}^{3}$ has ten coefficients (homogeneous polynomial of 4 variables). Therefore, a unique quadric $Q=0$ can be drawn through nine points in general position. Similarly, a pencil (one-parameter linear family) of quadrics $Q+\lambda Q^{\prime}=0$ can be drawn through eight points in general position, and a two-parameter linear family of quadrics $Q+\lambda Q^{\prime}+\mu Q^{\prime \prime}=0$ can be drawn through seven points in general position. Generically, solution of a system of three quadratic equations

$$
Q=0, \quad Q^{\prime}=0, \quad Q^{\prime \prime}=0
$$

for the intersection of three quadrics in $\mathbb{C P}^{3}$ consists of eight points. It can be shown that the three quadrics spanning the above-mentioned twoparameter family can be considered generic enough for such a conclusion. Clearly, the resulting eight points lie on every quadric of the two-parameter family.

Theorem 15 (Elementary hexahedron of a Q-net in a quadric) If seven points $f, f_{i}$, and $f_{i j}(1 \leq i<j \leq 3)$ of an elementary hexahedron of a $Q$-net $f: \mathbb{Z}^{m} \rightarrow \mathbb{R P}^{N}$ belong to a quadric $Q \subset \mathbb{R} \mathbb{P}^{N}$, then so does the eighth point $f_{123}$.

Proof. The original seven points can be considered lying in a three-dimensional space, and they are known to belong to three (degenerate) quadrics the pairs of planes $\Pi_{j k} \cup \tau_{i} \Pi_{j k}$ for $(j k)=(12),(23)$, (31). Clearly, the eighth intersection point of these quadrics is $f_{123}=\tau_{1} \Pi_{23} \cap \tau_{2} \Pi_{31} \cap \tau_{3} \Pi_{12}$, and this has to be the associated point. According to Theorem [14 it belongs to any quadric through the original seven points, in particular, to 2 .

\section{$3 \quad$ Geometries of spheres}

\subsection{Lie geometry}

A classical source on Lie geometry is Blaschke's book [B1], see also a modern account by Cecil [C].

Following geometric objects in the Euclidean space $\mathbb{R}^{N}$ are elements of Lie geometry:

- Oriented hyperspheres. A hypersphere in $\mathbb{R}^{N}$ with center $c \in \mathbb{R}^{N}$ and radius $r>0$ is described by the equation $S=\left\{x \in \mathbb{R}^{N}:|x-c|^{2}=r^{2}\right\}$. It divides $\mathbb{R}^{N}$ in two parts, inner and outer. If one denotes one of 
two parts of $\mathbb{R}^{N}$ as positive, one comes to the notion of an oriented hypersphere. Thus, there are two oriented hyperspheres $S^{ \pm}$for any $S$. One can take the orientation of a hypersphere into account by assigning a signed radius $\pm r$ to it. For instance, one can assign positive radii $r>0$ to hyperspheres with the inward field of unit normals and negative radii $r<0$ to hyperspheres with the outward field of unit normals.

- Oriented hyperplanes. A hyperplane in $\mathbb{R}^{N}$ is given by the equation $P=\left\{x \in \mathbb{R}^{N}:\langle v, x\rangle=d\right\}$, with a unit normal $v \in \mathbb{S}^{N-1}$ and $d \in \mathbb{R}$. Clearly, the pairs $(v, d)$ and $(-v,-d)$ represent one and the same hyperplane. It divides $\mathbb{R}^{N}$ in two halfspaces. Denoting one of two halfspaces as positive, one arrives at the notion of an oriented hyperplane. Thus, there are two oriented hyperplanes $P^{ \pm}$for any $P$. One can take the orientation of a hypersphere into account by assigning the pair $(v, d)$ to the hyperplane with the unit normal $v$ pointing into the positive halfspace.

- Points. One considers points $x \in \mathbb{R}^{N}$ as hyperspheres of a vanishing radius.

- Infinity. One compactifies the space $\mathbb{R}^{N}$ by adding the point at infinity $\infty$, with the understanding that a basis of open neighborhoods of $\infty$ is given, e.g., by the outer parts of the hyperspheres $|x|^{2}=r^{2}$. Topologically the so defined compactification is equivalent to a sphere $\mathbb{S}^{N}$.

- Contact elements. A contact element of a hypersurface is a pair consisting of a point $x \in \mathbb{R}^{N}$ and an (oriented) hyperplane $P$ through $x$; alternatively, one can use a normal vector $v$ to $P$ at $x$. In the framework of Lie geometry, a contact element can be identified with a set (a pencil) of all hyperspheres $S$ through $x$ which are in an oriented contact with $P$ (and with one another), thus sharing the normal vector $v$ at $x$, see Fig. 9 .

All these elements are modelled in Lie geometry as points, resp. lines, in the $(N+2)$-dimensional projective space $\mathbb{P}\left(\mathbb{R}^{N+1,2}\right)$ with the space of homogeneous coordinates $\mathbb{R}^{N+1,2}$. The latter is the space spanned by $N+3$ linearly independent vectors $\mathbf{e}_{1}, \ldots, \mathbf{e}_{N+3}$ and equipped with the pseudo- 


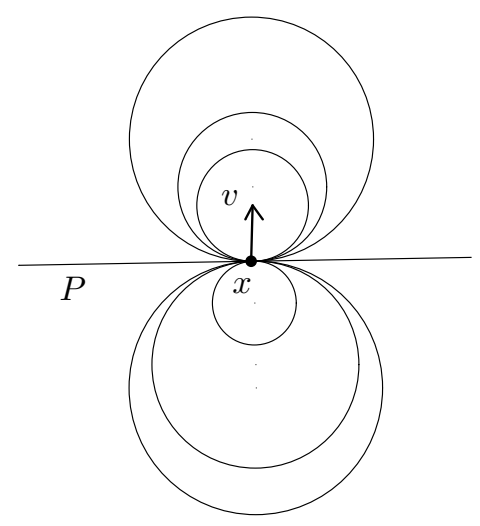

Figure 9: Contact element

euclidean scalar product

$$
\left\langle\mathbf{e}_{i}, \mathbf{e}_{j}\right\rangle=\left\{\begin{aligned}
1, & i=j \in\{1, \ldots, N+1\}, \\
-1, & i=j \in\{N+2, N+3\}, \\
0, & i \neq j .
\end{aligned}\right.
$$

It is convenient to introduce two isotropic vectors

$$
\mathbf{e}_{0}=\frac{1}{2}\left(\mathbf{e}_{N+2}-\mathbf{e}_{N+1}\right), \quad \mathbf{e}_{\infty}=\frac{1}{2}\left(\mathbf{e}_{N+2}+\mathbf{e}_{N+1}\right),
$$

for which

$$
\left\langle\mathbf{e}_{0}, \mathbf{e}_{0}\right\rangle=\left\langle\mathbf{e}_{\infty}, \mathbf{e}_{\infty}\right\rangle=0, \quad\left\langle\mathbf{e}_{0}, \mathbf{e}_{\infty}\right\rangle=-\frac{1}{2} .
$$

The models of the above elements in the space $\mathbb{R}^{N+1,2}$ of homogeneous coordinates are as follows:

- Oriented hypersphere with center $c \in \mathbb{R}^{N}$ and signed radius $r \in \mathbb{R}$ :

$$
\hat{s}=c+\mathbf{e}_{0}+\left(|c|^{2}-r^{2}\right) \mathbf{e}_{\infty}+r \mathbf{e}_{N+3} .
$$

- Oriented hyperplane $\langle v, x\rangle=d$ with $v \in \mathbb{S}^{N-1}$ and $d \in \mathbb{R}$ :

$$
\hat{p}=v+0 \cdot \mathbf{e}_{0}+2 d \mathbf{e}_{\infty}+\mathbf{e}_{N+3} .
$$

- Point $x \in \mathbb{R}^{N}$ :

$$
\hat{x}=x+\mathbf{e}_{0}+|x|^{2} \mathbf{e}_{\infty}+0 \cdot \mathbf{e}_{N+3} .
$$


- Infinity $\infty$ :

$$
\hat{\infty}=\mathbf{e}_{\infty}
$$

- Contact element $(x, P)$ :

$$
\operatorname{span}(\hat{x}, \hat{p}) .
$$

In the projective space $\mathbb{P}\left(\mathbb{R}^{N+1,2}\right)$ the first four types of elements are represented by the points which are equivalence classes of (47)-(7) with respect to the relation $\xi \sim \eta \Leftrightarrow \xi=\lambda \eta$ with $\lambda \in \mathbb{R}^{*}$ for $\xi, \eta \in \mathbb{R}^{N+1,2}$. A contact element is represented by the line in $\mathbb{P}\left(\mathbb{R}^{N+1,2}\right)$ through the points with the representatives $\hat{x}$ and $\hat{p}$. We mention several fundamentally important features of this model:

(i) All the above elements belong to the Lie quadric $\mathbb{P}\left(\mathbb{L}^{N+1,2}\right)$, where

$$
\mathbb{L}^{N+1,2}=\left\{\xi \in \mathbb{R}^{N+1,2}:\langle\xi, \xi\rangle=0\right\} .
$$

Moreover, points of $\mathbb{P}\left(\mathbb{L}^{N+1,2}\right)$ are in a one-to-one correspondence with oriented hyperspheres in $\mathbb{R}^{N}$, including degenerate case: proper hyperspheres correspond to points of $\mathbb{P}\left(\mathbb{L}^{N+1,2}\right)$ with both $\mathbf{e}_{0^{-}}$and $\mathbf{e}_{N+3}$-components non-vanishing, hyperplanes correspond to points of $\mathbb{P}\left(\mathbb{L}^{N+1,2}\right)$ with vanishing $\mathbf{e}_{0}$-component, points correspond to points of $\mathbb{P}\left(\mathbb{L}^{N+1,2}\right)$ with vanishing $\mathbf{e}_{N+3}$-component, and infinity corresponds to the only point of $\mathbb{P}\left(\mathbb{L}^{N+1,2}\right)$ with both $\mathbf{e}_{0^{-}}$and $\mathbf{e}_{N+3^{-c o m p o n e n t s}}$ vanishing.

(ii) Two oriented hyperspheres $S_{1}, S_{2}$ are in an oriented contact (i.e., are tangent to each other with the unit normals at tangency pointing in the same direction), if and only if

$$
\left|c_{1}-c_{2}\right|^{2}=\left(r_{1}-r_{2}\right)^{2},
$$

and this is equivalent to $\left\langle\hat{s}_{1}, \hat{s}_{2}\right\rangle=0$.

(iii) An oriented hypersphere $S=\left\{x \in \mathbb{R}^{N}:|x-c|^{2}=r^{2}\right\}$ is in an oriented contact with an oriented hyperplane $P=\left\{x \in \mathbb{R}^{N}:\langle v, x\rangle=d\right\}$, if and only if

$$
\langle c, v\rangle-r-d=0 .
$$

Indeed, equation of the hyperplane $P$ tangent to $S$ at $x_{0} \in S$ reads: $\left\langle x_{0}-c, x-c\right\rangle=r^{2}$. Denoting by $v=\left(c-x_{0}\right) / r$ the unit normal vector of $P$ (recall that the positive radii are assigned to spheres with inward unit normals), we can write the above equation as $\langle v, x\rangle=d$ 
with $d=\left\langle c,\left(c-x_{0}\right) / r\right\rangle-r=\langle c, v\rangle-r$, which proves eq. (11). Now, the latter equation is equivalent to $\langle\hat{s}, \hat{p}\rangle=0$.

(iv) A point $x$ can be considered as a hypersphere of radius $r=0$ (in this case both oriented hyperspheres coincide). An incidence relation $x \in S$ with a hypersphere $S$ (resp. $x \in P$ with a hyperplane $P$ ) can be interpreted as a particular case of an oriented contact of a sphere of radius $r=0$ with $S$ (resp. with $P$ ), and it takes place if and only if $\langle\hat{x}, \hat{s}\rangle=0$ (resp. $\langle\hat{x}, \hat{p}\rangle=0)$.

(v) For any hyperplane $P$, there holds $\langle\hat{\infty}, \hat{p}\rangle=0$. One can interpret hyperplanes as hyperspheres (of an infinite radius) through $\infty$. More precisely, a hyperplane $\langle v, x\rangle=d$ can be interpreted as a limit, as $r \rightarrow \infty$, of the hyperspheres of radii $r$ with the centers located at $c=r v+u$, with $\langle v, u\rangle=d$. Indeed, the representatives (4) of such spheres are

$$
\begin{aligned}
\hat{s} & =(r v+u)+\mathbf{e}_{0}+(2 d r+\langle u, u\rangle) \mathbf{e}_{\infty}+r \mathbf{e}_{N+3} \\
& \sim(v+O(1 / r))+(1 / r) \mathbf{e}_{0}+(2 d+O(1 / r)) \mathbf{e}_{\infty}+\mathbf{e}_{N+3} \\
& =\hat{p}+O(1 / r) .
\end{aligned}
$$

Moreover, for similar reasons, the infinity $\infty$ can be considered as a limiting position of any sequence of points $x$ with $|x| \rightarrow \infty$.

(vi) Any two hyperspheres $S_{1}, S_{2}$ in an oriented contact determine a contact element (their point of contact and their common tangent hyperplane). For their representatives $\hat{s}_{1}, \hat{s}_{2}$ in $\mathbb{R}^{N+1,2}$, the line in $\mathbb{P}\left(\mathbb{R}^{N+1,2}\right)$ through the corresponding points in $\mathbb{P}\left(\mathbb{L}^{N+1,2}\right)$ is isotropic, i.e., lies entirely on the Lie quadric $\mathbb{P}\left(\mathbb{L}^{N+1,2}\right)$. This follows from

$$
\left\langle\alpha_{1} \hat{s}_{1}+\alpha_{2} \hat{s}_{2}, \alpha_{1} \hat{s}_{1}+\alpha_{2} \hat{s}_{2}\right\rangle=2 \alpha_{1} \alpha_{2}\left\langle\hat{s}_{1}, \hat{s}_{2}\right\rangle=0 .
$$

Such a line contains exactly one point whose representative $\hat{x}$ has vanishing $\mathbf{e}_{N+3}$-component (and corresponds to $x$, the common point of contact of all hyperspheres), and, if $x \neq \infty$, exactly one point whose representative $\hat{p}$ has vanishing $\mathbf{e}_{0}$-component (and corresponds to $P$, the common tangent hyperplane of all hyperspheres). In case when an isotropic line contains $\hat{\infty}$, all its points represent parallel hyperplanes, which constitute a contact element through $\infty$.

Thus, if one considers hyperplanes as hyperspheres of infinite radii, and points as hyperspheres of vanishing radii, then one can conclude that: 
- oriented hyperspheres are in a one-to-one correspondence with points of the Lie quadric $\mathbb{P}\left(\mathbb{L}^{N+1,2}\right)$ in the projective space $\mathbb{P}\left(\mathbb{R}^{N+1,2}\right)$;

- oriented contact of two oriented hyperspheres corresponds to orthogonality of (any) representatives of the corresponding points in $\mathbb{P}\left(\mathbb{R}^{N+1,2}\right)$.

- contact elements of hypersurfaces are in a one-to-one correspondence with isotropic lines in $\mathbb{P}\left(\mathbb{R}^{N+1,2}\right)$. We will denote the set of all such lines by $\mathcal{L}_{0}^{N+1,2}$.

According to F. Klein's Erlangen Program, Lie geometry is the study of properties of transformations which map oriented hyperspheres (including points and hyperplanes) to oriented hyperspheres and, moreover, preserve the oriented contact of hypersphere pairs. In the projective model described above, Lie geometry is the study of projective transformations of $\mathbb{P}\left(\mathbb{R}^{N+1,2}\right)$ which leave $\mathbb{P}\left(\mathbb{L}^{N+1,2}\right)$ invariant, and, moreover, preserve orthogonality of points of $\mathbb{P}\left(\mathbb{L}^{N+1,2}\right)$ (which is understood as orthogonality of their lifts to $\mathbb{L}^{N+1,2} \subset \mathbb{R}^{N+1,2}$; clearly, this relation does not depend on the choice of lifts). Such transformations are called Lie sphere transformations.

\section{Theorem 16 (Fundamental theorem of Lie geometry)}

a) The group of Lie sphere transformations is isomorphic to $O(N+1,2) /\{ \pm I\}$.

b) Every line preserving diffeomorphism of $\mathbb{P}\left(\mathbb{L}^{N+1,2}\right)$ is the restriction to $\mathbb{P}\left(\mathbb{L}^{N+1,2}\right)$ of a Lie sphere transformation.

Since (non-)vanishing of the $\mathbf{e}_{0^{-}}$or of the $\mathbf{e}_{N+3^{-}}$-component of a point in $\mathbb{P}\left(\mathbb{L}^{N+1,2}\right)$ is not invariant under a general Lie sphere transformation, there is no distinction between oriented hyperspheres, oriented hyperplanes and points in Lie geometry.

\subsection{Möbius geometry}

Blaschke's book B1] serves also as a classical source on Möbius geometry, a modern account can be found in $\mathrm{HJ}]$.

Möbius geometry is a subgeometry of Lie geometry, with points distinguishable among all hyperspheres as those of radius zero. Thus, Möbius geometry studies properties of hyperspheres invariant under the subgroup of Lie sphere transformations preserving the set of points. In the projective model, points of $\mathbb{R}^{N}$ are distinguished as points of $\mathbb{P}\left(\mathbb{L}^{N+1,2}\right)$ with the vanishing $\mathbf{e}_{N+3}$-component. (Of course, one could replace here $\mathbf{e}_{N+3}$ by any time-like vector.) Thus, Möbius geometry studies the subgroup of Lie 
sphere transformations preserving the subset of $\mathbb{P}\left(\mathbb{L}^{N+1,2}\right)$ with the vanishing $\mathbf{e}_{N+3}$-component. Following geometric objects in $\mathbb{R}^{N}$ are elements of Möbius geometry.

- (Non-oriented) hyperspheres $S=\left\{x \in \mathbb{R}^{N}:|x-c|^{2}=r^{2}\right\}$ with centers $c \in \mathbb{R}^{N}$ and radii $r>0$.

- (Non-oriented) hyperplanes $P=\left\{x \in \mathbb{R}^{N}:\langle v, x\rangle=d\right\}$, with unit normals $v \in \mathbb{S}^{N-1}$ and $d \in \mathbb{R}$.

- Points $x \in \mathbb{R}^{N}$.

- Infinity $\infty$ which compactifies $\mathbb{R}^{N}$ into $\mathbb{S}^{N}$.

In modelling these elements, one can use the Lie-geometric description and just omit the $\mathbf{e}_{N+3}$-component. The resulting objects are points of the $(N+1)$-dimensional projective space $\mathbb{P}\left(\mathbb{R}^{N+1,1}\right)$ with the space of homogeneous coordinates $\mathbb{R}^{N+1,1}$. The latter is the space spanned by $N+2$ linearly independent vectors $\mathbf{e}_{1}, \ldots, \mathbf{e}_{N+2}$ and equipped with the Minkowski scalar product

$$
\left\langle\mathbf{e}_{i}, \mathbf{e}_{j}\right\rangle=\left\{\begin{aligned}
1, & i=j \in\{1, \ldots, N+1\}, \\
-1, & i=j=N+2, \\
0, & i \neq j .
\end{aligned}\right.
$$

We continue to use notations (3) in the context of the Möbius geometry. The above elements are modelled in the space $\mathbb{R}^{N+1,1}$ of homogeneous coordinates as follows:

- Hypersphere with center $c \in \mathbb{R}^{N}$ and radius $r>0$ :

$$
\hat{s}=c+\mathbf{e}_{0}+\left(|c|^{2}-r^{2}\right) \mathbf{e}_{\infty} .
$$

- Hyperplane $\langle v, x\rangle=d$ with $v \in \mathbb{S}^{N-1}$ and $d \in \mathbb{R}$ :

$$
\hat{p}=v+0 \cdot \mathbf{e}_{0}+2 d \mathbf{e}_{\infty}
$$

- Point $x \in \mathbb{R}^{N}$ :

$$
\hat{x}=x+\mathbf{e}_{0}+|x|^{2} \mathbf{e}_{\infty} .
$$

- Infinity $\infty$ :

$$
\hat{\infty}=\mathbf{e}_{\infty} .
$$


In the projective space $\mathbb{P}\left(\mathbb{R}^{N+1,1}\right)$ these elements are represented by points which are equivalence classes of (12) -15) with respect to the usual relation $\xi \sim \eta \Leftrightarrow \xi=\lambda \eta$ with $\lambda \in \mathbb{R}^{*}$ for $\xi, \eta \in \mathbb{R}^{N+1,1}$. Fundamental features of these identifications:

(i) The infinity $\hat{\infty}$ can be considered as a limit of any sequence of $\hat{x}$ for $x \in \mathbb{R}^{N}$ with $|x| \rightarrow \infty$. Elements $x \in \mathbb{R}^{N} \cup\{\infty\}$ are in a one-to-one correspondence with points of the projectivized light cone $\mathbb{P}\left(\mathbb{L}^{N+1,1}\right)$, where

$$
\mathbb{L}^{N+1,1}=\left\{\xi \in \mathbb{R}^{N+1,1}:\langle\xi, \xi\rangle=0\right\} .
$$

Points $x \in \mathbb{R}^{N}$ correspond to points of $\mathbb{P}\left(\mathbb{L}^{N+1,1}\right)$ with a non-vanishing $\mathbf{e}_{0}$-component, while $\infty$ corresponds to the only point of $\mathbb{P}\left(\mathbb{L}^{N+1,1}\right)$ with the vanishing $\mathbf{e}_{0}$-component.

(ii) Hyperspheres $\hat{s}$ and hyperplanes $\hat{p}$ belong to $\mathbb{P}\left(\mathbb{R}_{\text {out }}^{N+1,1}\right)$, where

$$
\mathbb{R}_{\text {out }}^{N+1,1}=\left\{\xi \in \mathbb{R}^{N+1,1}:\langle\xi, \xi\rangle>0\right\}
$$

is the set of space-like vectors of the Minkowski space $\mathbb{R}^{N+1,1}$. Hyperplanes can be interpreted as hyperspheres (of an infinite radius) through $\infty$.

(iii) Two hyperspheres $S_{1}, S_{2}$ with centers $c_{1}, c_{2}$ and radii $r_{1}, r_{2}$ intersect orthogonally, if and only if

$$
\left|c_{1}-c_{2}\right|^{2}=r_{1}^{2}+r_{2}^{2},
$$

which is equivalent to $\left\langle\hat{s}_{1}, \hat{s}_{2}\right\rangle=0$. Similarly, a hypersphere $S$ intersects orthogonally with a hyperplane $P$, if and only if its center lies in $P$ :

$$
\langle c, v\rangle-d=0,
$$

which is equivalent to $\langle\hat{s}, \hat{p}\rangle=0$.

(iv) A point $x$ can be considered as a limiting case of a hypersphere with radius $r=0$. An incidence relation $x \in S$ with a hypersphere $S$ (resp. $x \in P$ with a hyperplane $P$ ) can be interpreted as a particular case of an orthogonal intersection of a sphere of radius $r=0$ with $S$ (resp. with $P$ ), and it takes place if and only if $\langle\hat{x}, \hat{s}\rangle=0($ resp. $\langle\hat{x}, \hat{p}\rangle=0)$.

Note that a hypersphere $S$ can also be interpreted as the set of points $x \in S$. Correspondingly, it admits, along with the representation $\hat{s}$, the dual representation as a transversal intersection of $\mathbb{P}\left(\mathbb{L}^{N+1,1}\right)$ with the projective 
$N$-space $\mathbb{P}\left(\hat{s}^{\perp}\right)$, polar to the point $\hat{s}$ with respect to $\mathbb{P}\left(\mathbb{L}^{N+1,1}\right)$; here, of course, $\hat{s}^{\perp}=\left\{\hat{x} \in \mathbb{R}^{N+1,1}:\langle\hat{s}, \hat{x}\rangle=0\right\}$. This can be generalized to model lower-dimensional spheres.

- Spheres. A $k$-sphere is a (generic) intersection of $N-k$ hyperspheres $S_{i}$ $(i=1, \ldots, N-k)$. The intersection of $N-k$ hyperspheres represented by $\hat{s}_{i} \in \mathbb{R}_{\text {out }}^{N+1,1}(i=1, \ldots, N-k)$ is generic if the $(N-k)$-dimensional linear subspace of $\mathbb{R}^{N+1,1}$ spanned by $\hat{s}_{i}$ is space-like:

$$
\Sigma=\operatorname{span}\left(\hat{s}_{1}, \ldots, \hat{s}_{N-k}\right) \subset \mathbb{R}_{\text {out }}^{N+1,1} .
$$

As a set of points, this $k$-sphere is represented as $\mathbb{P}\left(\mathbb{L}^{N+1,1} \cap \Sigma^{\perp}\right.$, where

$$
\Sigma^{\perp}=\bigcap_{i=1}^{N-k} \hat{s}_{i}^{\perp}=\left\{\hat{x} \in \mathbb{R}^{N+1,1}:\left\langle\hat{s}_{1}, \hat{x}\right\rangle=\ldots=\left\langle\hat{s}_{N-k}, \hat{x}\right\rangle=0\right\}
$$

is a $(k+2)$-dimensional linear subspace of $\mathbb{R}^{N+1,1}$ of signature $(k+1,1)$. Through any $k+2$ points $x_{1}, \ldots, x_{k+2} \in \mathbb{R}^{N}$ in general position one can draw a unique $k$-sphere. It corresponds to the $(k+2)$-dimensional linear subspace

$$
\Sigma^{\perp}=\operatorname{span}\left(\hat{x}_{1}, \ldots, \hat{x}_{k+2}\right),
$$

of signature $(k+1,1)$, with $k+2$ linearly independent isotropic vectors $\hat{x}_{1}, \ldots, \hat{x}_{k+2} \in \mathbb{L}^{N+1,1}$. In the polar formulation, this $k$-sphere corresponds to the $(N-k)$-dimensional space-like linear subspace

$$
\Sigma=\bigcap_{i=1}^{k+2} \hat{x}_{i}^{\perp}=\left\{\hat{s} \in \mathbb{R}^{N+1,1}:\left\langle\hat{s}, \hat{x}_{1}\right\rangle=\ldots=\left\langle\hat{s}, \hat{x}_{k+2}\right\rangle=0\right\} .
$$

Möbius geometry is the study of properties of (non)-oriented hyperspheres invariant with respect to projective transformations of $\mathbb{P}\left(\mathbb{R}^{N+1,1}\right)$ which map points to points, i.e., which leave $\mathbb{P}\left(\mathbb{L}^{N+1,1}\right)$ invariant. Such transformations are called Möbius transformations.

\section{Theorem 17 (Fundamental theorem of Möbius geometry)}

a) The group of Möbius transformations is isomorphic to $O(N+1,1) /\{ \pm I\} \simeq$ $O^{+}(N+1,1)$, the group of Lorentz transformations of $\mathbb{R}^{N+1,1}$ preserving the time-like direction.

b) Every conformal diffeomorphism of $\mathbb{S}^{N} \simeq \mathbb{R}^{N} \cup\{\infty\}$ is induced by the restriction to $\mathbb{P}\left(\mathbb{L}^{N+1,1}\right)$ of a Möbius transformation. 
The group $O^{+}(N+1,1)$ is generated by reflections

$$
A_{\hat{s}}: \mathbb{R}^{N+1,1} \rightarrow \mathbb{R}^{N+1,1}, \quad A_{\hat{s}}(\hat{x})=\hat{x}-\frac{2\langle\hat{s}, \hat{x}\rangle}{\langle\hat{s}, \hat{s}\rangle} \hat{s} .
$$

If $\hat{s}$ is a hypersphere (12), then the transformation induced on $\mathbb{R}^{N}$ by $A_{\hat{s}}$ is obtained from (20) by a computation with the representatives (14) for points and is given by:

$$
x \mapsto c+\frac{r^{2}}{|x-c|^{2}}(x-c)
$$

(inversion in the hypersphere $S=\left\{x \in \mathbb{R}^{N}:|x-c|^{2}=r^{2}\right\}$ ); similarly, if $\hat{s}=\hat{p}$ is the hyperplane (13), then the transformation induced on $\mathbb{R}^{N}$ by $A_{\hat{p}}$ is easily computed to be

$$
x \mapsto x-\frac{2(\langle v, x\rangle-d)}{\langle v, v\rangle} v
$$

(reflection in the hyperplane $P=\left\{x \in \mathbb{R}^{N}:\langle v, x\rangle=d\right\}$ ).

Since (non-)vanishing of the $\mathbf{e}_{\infty}$-component of a point in $\mathbb{P}\left(\mathbb{R}^{N+1,1}\right)$ is not invariant under a general Möbius transformation, there is no distinction in Möbius geometry between hyperspheres and hyperplanes.

\subsection{Laguerre geometry}

Also in the case of Laguerre geometry the Blaschke's book B1 serves as the indispensable classical source. One can find a modern account, e.g., in Benz, C, PP.

Laguerre geometry is a subgeometry of Lie geometry, with hyperplanes distinguishable among all hyperspheres, as hyperspheres through $\infty$. Thus, Laguerre geometry studies properties of hyperspheres invariant under the subgroup of Lie sphere transformations which preserve the set of hyperplanes. Following objects in $\mathbb{R}^{N}$ are elements of the Laguerre geometry.

- (Oriented) hyperspheres $S=\left\{x \in \mathbb{R}^{N}:|x-c|^{2}=r^{2}\right\}$ with centers $c \in \mathbb{R}^{N}$ and signed radii $r \in \mathbb{R}$, can be put into correspondence with $(N+1)$-tuples $(c, r)$.

- Points $x \in \mathbb{R}^{N}$ are considered as hyperspheres of radius zero, and are put into correspondence with $(N+1)$-tuples $(x, 0)$. 
- (Oriented) hyperplanes $P=\left\{x \in \mathbb{R}^{N}:\langle v, x\rangle=d\right\}$, with unit normals $v \in \mathbb{S}^{N-1}$ and $d \in \mathbb{R}$, can be put into correspondence with $(N+1)$ tuples $(v, d)$.

In the projective model of Lie geometry, hyperplanes are distinguished as elements of $\mathbb{P}\left(\mathbb{L}^{N+1,2}\right)$ with the vanishing $\mathbf{e}_{0}$-component. (Of course, one could replace here $\mathbf{e}_{0}$ by any isotropic vector.) Thus, Laguerre geometry studies the subgroup of Lie sphere transformations preserving the subset of $\mathbb{P}\left(\mathbb{L}^{N+1,2}\right)$ with the vanishing $\mathbf{e}_{0}$-component.

There seems to exist no model of Laguerre geometry, where hyperspheres and hyperplanes would be modelled as points of one and the same space. Depending on which of both types of elements is modelled by points, one comes to the Blaschke cylinder model or to the cyclographic model of Laguerre geometry. We will use the first of these models which has an advantage of a simpler description of the distinguished objects of the Laguerre geometry, which are hyperplanes. The main advantage of the second model is a simpler description of the group of Laguerre transformations.

The scene of the both models consists of two $(N+1)$-dimensional projective spaces, whose spaces of homogeneous coordinates, $\mathbb{R}^{N, 1,1}$ and $\left(\mathbb{R}^{N, 1,1}\right)^{*}$, are dual to one another and arise from $\mathbb{R}^{N+1,2}$ by "forgetting" the $\mathbf{e}_{0^{-}}$, resp. $\mathbf{e}_{\infty}$-components. Thus, $\mathbb{R}^{N, 1,1}$ is spanned by $N+2$ linearly independent vectors $\mathbf{e}_{1}, \ldots, \mathbf{e}_{N}, \mathbf{e}_{N+3}, \mathbf{e}_{\infty}$, and is equipped with a degenerate bilinear form of the signature $(N, 1,1)$ in which the above vectors are pairwise orthogonal, the first $N$ being space-like: $\left\langle\mathbf{e}_{i}, \mathbf{e}_{i}\right\rangle=1$ for $1 \leq i \leq N$, while the last two being time-like and isotropic, respectively: $\left\langle\mathbf{e}_{N+3}, \mathbf{e}_{N+3}\right\rangle=-1$ and $\left\langle\mathbf{e}_{\infty}, \mathbf{e}_{\infty}\right\rangle=0$. Similarly, $\left(\mathbb{R}^{N, 1,1}\right)^{*}$ is assumed to have an orthogonal basis consisting of $\mathbf{e}_{1}, \ldots, \mathbf{e}_{N}, \mathbf{e}_{N+3}, \mathbf{e}_{0}$, again with an isotropic last vector: $\left\langle\mathbf{e}_{0}, \mathbf{e}_{0}\right\rangle=0$. Note that one and the same symbol $\langle\cdot, \cdot\rangle$ is used to denote two degenerate bilinear forms in our two spaces. We will overload this symbol even more and use it also for the (non-degenerate) pairing between these two spaces, which is established by setting $\left\langle\mathbf{e}_{0}, \mathbf{e}_{\infty}\right\rangle=-\frac{1}{2}$, additionally to the above relations. (Note that a degenerate bilinear form cannot be used to identify a vector space with its dual.)

In both models mentioned above there holds:

- Hyperplane $P=(v, d)$ is modelled as a point in the space $\mathbb{P}\left(\mathbb{R}^{N, 1,1}\right)$ with a representative

$$
\hat{p}=v+2 d \mathbf{e}_{\infty}+\mathbf{e}_{N+3} .
$$


- Hypersphere $S=(c, r)$ is modelled as a point in the space $\mathbb{P}\left(\left(\mathbb{R}^{N, 1,1}\right)^{*}\right)$ with a representative

$$
\hat{s}=c+\mathbf{e}_{0}+r \mathbf{e}_{N+3} .
$$

Each one of the models appears if one considers one of the spaces as a preferred (fundamental) one, and interprets the points of the second space as hyperplanes in the preferred one. In the Blaschke cylinder model, the preferred space is the space $\mathbb{P}\left(\mathbb{R}^{N, 1,1}\right)$ whose points model hyperplanes $P \subset \mathbb{R}^{N}$. A hypersphere $S \subset \mathbb{R}^{N}$ is then modelled as a hyperplane $\left\{\xi \in \mathbb{P}\left(\mathbb{R}^{N, 1,1}\right)\right.$ : $\langle\hat{s}, \xi\rangle=0\}$ in the space $\mathbb{P}\left(\mathbb{R}^{N, 1,1}\right)$. Basic features of this model:

(i) Oriented hyperplanes $P \subset \mathbb{R}^{N}$ are in a one-to-one correspondence with points $\hat{p}$ of the quadric $\mathbb{P}\left(\mathbb{L}^{N, 1,1}\right)$, where

$$
\mathbb{L}^{N, 1,1}=\left\{\xi \in \mathbb{R}^{N, 1,1}:\langle\xi, \xi\rangle=0\right\} .
$$

(iii) Two oriented hyperplanes $P_{1}, P_{2} \subset \mathbb{R}^{N}$ are in an oriented contact (parallel), if and only if their representatives $\hat{p}_{1}, \hat{p}_{2}$ differ by a vector parallel to $\mathbf{e}_{\infty}$.

(iii) An oriented hypersphere $S \subset \mathbb{R}^{N}$ is in an oriented contact with an oriented hyperplane $P \subset \mathbb{R}^{N}$, if and only if $\hat{p} \in \hat{s}$, that is, if $\langle\hat{p}, \hat{s}\rangle=$ 0 . Thus, a hypersphere $S$ is interpreted as a set of all its tangent hyperplanes.

The quadric $\mathbb{P}\left(\mathbb{L}^{N, 1,1}\right)$ is diffeomorphic to the Blaschke cylinder

$$
\mathcal{Z}=\left\{(v, d) \in \mathbb{R}^{N+1}:|v|=1\right\}=\mathbb{S}^{N-1} \times \mathbb{R} \subset \mathbb{R}^{N+1} .
$$

Two points of this cylinder represent parallel hyperplanes, if they lie on one straight line generator of $Z$ parallel to its axis. In the ambient space $\mathbb{R}^{N+1}$ of the Blaschke cylinder, oriented hyperspheres $S \subset \mathbb{R}^{N}$ are in a one-to-one correspondence with hyperplanes non-parallel to the axis of $Z$ :

$$
S \sim\left\{(v, d) \in \mathbb{R}^{N+1}:\langle c, v\rangle-d-r=0\right\} .
$$

An intersection of such a hyperplane with $Z$ consists of points in $Z$ which represent tangent hyperplanes to $S \subset \mathbb{R}^{N}$, as follows from eq. (11).

In this paper, we will not use the cyclographic model of Laguerre geometry; its short description is put in Appendix $\mathrm{A}$. 


\section{Discrete curvature line parametrization in Lie, Möbius and Laguerre geometries}

Starting from here, we restrict ourselves to the geometry of surfaces in the three-dimensional Euclidean space $\mathbb{R}^{3}$. Accordingly, one should set $N=3$ in all previous considerations.

It is natural to consider following objects as discrete surfaces in the various geometries discussed above:

- In Lie geometry, a surface is viewed as built of its contact elements. These contact elements are interpreted as points of the surface and tangent planes (or, equivalently, normals) at these points. This can be discretized in a natural way: a discrete surface is a map

$$
(x, P): \mathbb{Z}^{2} \rightarrow\left\{\text { contact elements of surfaces in } \mathbb{R}^{3}\right\},
$$

or, in the projective model of Lie geometry, a map

$$
\ell: \mathbb{Z}^{2} \rightarrow \mathcal{L}_{0}^{4,2}
$$

where, recall, $\mathcal{L}_{0}^{4,2}$ denotes the set of isotropic lines in $\mathbb{P}\left(\mathbb{R}^{4,2}\right)$.

- In Möbius geometry, a surface is viewed simply as built of points. A discrete surface is a map

$$
x: \mathbb{Z}^{2} \rightarrow \mathbb{R}^{3},
$$

or, in the projective model, a map

$$
\hat{x}: \mathbb{Z}^{2} \rightarrow \mathbb{P}\left(\mathbb{L}^{4,1}\right) .
$$

- In Laguerre geometry, a surface is viewed as the envelope of the system of its tangent planes. A discrete surface is a map

$$
\left.P: \mathbb{Z}^{2} \rightarrow \text { oriented planes in } \mathbb{R}^{3}\right\},
$$

or, in the projective model, a map

$$
\hat{p}: \mathbb{Z}^{2} \rightarrow \mathbb{P}\left(\mathbb{L}^{3,1,1}\right) .
$$

It should be mentioned that a substantial part of the description of a surface in Laguerre geometry is its Gauss map

$$
v: \mathbb{Z}^{2} \rightarrow \mathbb{S}^{2}
$$

consisting of unit normals $v$ to the tangent planes $P=(v, d)$.

Thus, description of a discrete surface in Lie geometry contains more information than description of a discrete surface in Möbius or in Laguerre geometry. Actually, the former merges the two latter ones. 


\subsection{Lie geometry}

The following definition is a discretization of the Lie-geometric description of curvature line parametrized surfaces, as found, e.g., in [B1].

\section{Definition 18 (Principal contact element nets. Euclidean model)} A map

$$
(x, P): \mathbb{Z}^{2} \rightarrow\left\{\text { contact elements of surfaces in } \mathbb{R}^{3}\right\}
$$

is called a principal contact element net, if any two neighboring contact elements $(x, P),\left(x_{i}, P_{i}\right)$ have a sphere $S^{(i)}$ in common, that is, a sphere touching both planes $P, P_{i}$ at the corresponding points $x, x_{i}$.

Thus, the normals to the neighboring planes $P, P_{i}$ at the corresponding points $x, x_{i}$ intersect at a point $c^{(i)}$ (the center of the sphere $S^{(i)}$ ), and the distances from $c^{(i)}$ to $x$ and to $x_{i}$ are equal, see Fig. 10. The spheres $S^{(i)}$, attached to the edges of $\mathbb{Z}^{2}$ parallel to the $i$-th coordinate axis, will be called principal curvature spheres of the discrete surface.

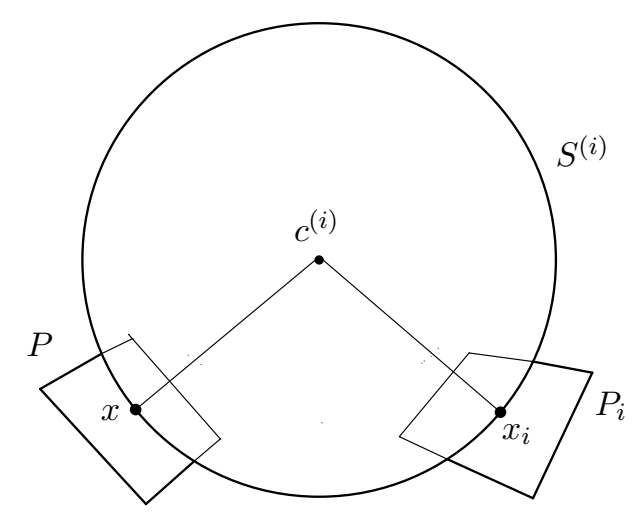

Figure 10: Principal curvature sphere

A direct translation of Definition [18 into the projective model looks as follows:

Definition 19 (Principal contact element nets. Projective model) A map $\ell: \mathbb{Z}^{2} \rightarrow \mathcal{L}_{0}^{4,2}$ is called a principal contact element net, if it is a 
discrete congruence of isotropic lines in $\mathbb{P}\left(\mathbb{R}^{4,2}\right)$, that is, any two neighboring lines intersect:

$$
\ell(u) \cap \ell\left(u+e_{i}\right)=\hat{s}^{(i)}(u) \in \mathbb{P}\left(\mathbb{L}^{4,2}\right), \quad \forall u \in \mathbb{Z}^{2}, \quad \forall i=1,2 .
$$

In the projective model, the representatives of the principal curvature spheres $S^{(i)}$ of the $i$-th coordinate direction build the corresponding focal net of the line congruence $\ell$;

$$
\hat{s}^{(i)}: \mathbb{Z}^{2} \rightarrow \mathbb{P}\left(\mathbb{L}^{4,2}\right), \quad i=1,2,
$$

cf. Definition [1]. According to Theorem [12, both focal nets are Q-nets in $\mathbb{P}\left(\mathbb{R}^{4,2}\right)$. This motivates the following definition.

\section{Definition 20 (Discrete R-congruence of spheres) A map}

$$
S: \mathbb{Z}^{m} \rightarrow\left\{\text { oriented spheres in } \mathbb{R}^{3}\right\}
$$

is called a discrete $R$-congruence (Ribaucour congruence) of spheres, if the corresponding map

$$
\hat{s}: \mathbb{Z}^{m} \rightarrow \mathbb{P}\left(\mathbb{L}^{4,2}\right)
$$

is a $Q$-net in $\mathbb{P}\left(\mathbb{R}^{4,2}\right)$.

A geometric characterization of discrete R-congruences will be given in Sect. 5 .

Corollary 21 (Curvature spheres build an R-congruence) For a discrete contact element net, the principal curvature spheres of the $i$-th coordinate direction $(i=1,2)$ build a two-dimensional discrete $R$-congruence.

Turning to transformations of principal contact element nets, we introduce the following definition.

Definition 22 (Ribaucour transformation. Euclidean model) Two principal contact element nets

$$
(x, P),\left(x^{+}, P^{+}\right): \mathbb{Z}^{2} \rightarrow\left\{\text { contact elements of surfaces in } \mathbb{R}^{3}\right\}
$$

are called Ribaucour transforms of one another, if any two corresponding contact elements $(x, P)$ and $\left(x^{+}, P^{+}\right)$have a sphere $S$ in common, that is, a sphere which touches both planes $P, P^{+}$at the corresponding points $x, x^{+}$.

Again, a direct translation of Definition 22 into the language of the projective model gives: 


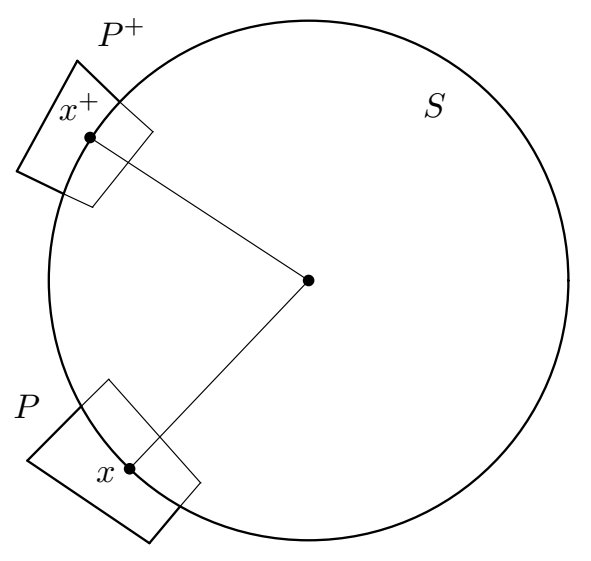

Figure 11: Ribaucour transformation

Definition 23 (Ribaucour transformation. Projective model) Two principal contact element nets $\ell, \ell^{+}: \mathbb{Z}^{2} \rightarrow \mathcal{L}_{0}^{4,2}$ are called Ribaucour transforms of one another, if these discrete congruences of isotropic lines are in the relation of F-transformation, that is, if any pair of the corresponding lines intersect:

$$
\ell(u) \cap \ell^{+}(u)=\hat{s}(u) \in \mathbb{P}\left(\mathbb{L}^{4,2}\right), \quad \forall u \in \mathbb{Z}^{2} .
$$

Spheres $S$ of a Ribaucour transformation are attached to the vertices $u$ of the lattice $\mathbb{Z}^{2}$, or, better, to the "vertical" edges connecting the vertices $(u, 0)$ and $(u, 1)$ of the lattice $\mathbb{Z}^{2} \times\{0,1\}$. In the projective model, their representatives

$$
\hat{s}: \mathbb{Z}^{2} \rightarrow \mathbb{P}\left(\mathbb{L}^{4,2}\right)
$$

build the focal net of the three-dimensional line congruence for the third coordinate direction. From Theorem 12 there follows:

Corollary 24 (Spheres of a Ribaucour transformation build an Rcongruence) The spheres of a generic Ribaucour transformation build a discrete R-congruence.

Now, we turn to the study of the geometry of an elementary quadrilateral of contact elements of a principal contact element net, consisting of $\ell \sim$ $(x, P), \ell_{1} \sim\left(x_{1}, P_{1}\right), \ell_{2} \sim\left(x_{2}, P_{2}\right)$, and $\ell_{12} \sim\left(x_{12}, P_{12}\right)$. 
We leave aside a degenerate umbilic situation, when all four lines have a common point and span a four-dimensional space. Geometrically, this means that one is dealing with four contact elements of a sphere $S \subset \mathbb{R}^{3}$. In this situation, one cannot draw any further conclusion about the four points $x, x_{1}, x_{2}, x_{12}$ on the sphere $S$ : they can be arbitrary.

In the non-umbilic situation, the space spanned by the four lines $\ell, \ell_{1}, \ell_{2}, \ell_{12}$ is three-dimensional. The four elements $\hat{x}, \hat{x}_{1}, \hat{x}_{2}, \hat{x}_{12} \in \mathbb{P}\left(\mathbb{L}^{4,2}\right)$ corresponding to the points $x, x_{1}, x_{2}, x_{12} \in \mathbb{R}^{3}$ are obtained as the intersection of the four isotropic lines $\ell, \ell_{1}, \ell_{2}, \ell_{12}$ with the projective hyperplane $\mathbb{P}\left(\mathbf{e}_{6}^{\perp}\right)$ in $\mathbb{P}\left(\mathbb{R}^{4,2}\right)$. Therefore, the four elements $\hat{x}, \hat{x}_{1}, \hat{x}_{2}, \hat{x}_{12}$ lie in a plane. A suitable framework for the study of this configuration is the projective model of the Möbius geometry. Namely, omitting the inessential (vanishing) $\mathbf{e}_{6}{ }^{-}$ component, we arrive at a planar quadrilateral in the Möbius sphere $\mathbb{P}\left(\mathbb{L}^{4,1}\right)$. We devote Sect. 4.2 to the study of such objects.

Analogously, the four elements $\hat{p}, \hat{p}_{1}, \hat{p}_{2}, \hat{p}_{12} \in \mathbb{P}\left(\mathbb{L}^{4,2}\right)$ corresponding to the planes $P, P_{1}, P_{2}, P_{12} \in \mathbb{R}^{3}$ are obtained as the intersection of the four isotropic lines $\ell, \ell_{1}, \ell_{2}, \ell_{12}$ with the projective hyperplane $\mathbb{P}\left(\mathbf{e}_{\infty}^{\perp}\right)$ in $\mathbb{P}\left(\mathbb{R}^{4,2}\right)$. Therefore, also the four elements $\hat{p}, \hat{p}_{1}, \hat{p}_{2}, \hat{p}_{12}$ lie in a plane. A suitable framework for the study of such a configuration is the projective model of the Laguerre geometry; this will be performed in Sect. 4.3 .

\subsection{Möbius geometry: circular nets}

Circular nets were introduced and studied in the context of integrable systems in [B, [CDS, KS.

Caution: in this section, the notation $\hat{x}$ refers to the Möbius-geometric representatives in $\mathbb{L}^{4,1}$, and not to the Lie-geometric ones in $\mathbb{L}^{4,2}$. The former are obtained from the latter one by omitting the (vanishing) $\mathbf{e}_{6}$-component.

We assume that the principal contact element nets under consideration are generic, i.e., do not contain umbilic quadruples. The main result of this section is the following claim.

\section{Theorem 25 (Points of principal contact element nets form circu-} lar nets) For a principal contact element net

$$
(x, P): \mathbb{Z}^{2} \rightarrow\left\{\text { contact elements of surfaces in } \mathbb{R}^{3}\right\},
$$

its points $x: \mathbb{Z}^{2} \rightarrow \mathbb{R}^{3}$ form a circular net.

This statement refers to the notion, which can be defined in two different ways. 
Definition 26 (Circular net. Euclidean model) A net $x: \mathbb{Z}^{m} \rightarrow \mathbb{R}^{3}$ is called circular, if the vertices of any elementary quadrilateral $\left(x, x_{i}, x_{i j}, x_{j}\right)$ (at any $u \in \mathbb{Z}^{m}$ and for all pairs $1 \leq i \neq j \leq m$ ) lie on a circle (in particular, are co-planar).

Definition 27 (Circular net. Projective model) $A$ net $x: \mathbb{Z}^{m} \rightarrow \mathbb{R}^{3}$ is called circular, if the corresponding $\hat{x}: \mathbb{Z}^{m} \rightarrow \mathbb{P}\left(\mathbb{L}^{4,1}\right)$ is a $Q$-net in $\mathbb{P}\left(\mathbb{R}^{4,1}\right)$.

This time a translation between the Euclidean model and the projective model is not straightforward and actually constitutes the matter of Theorem 25] indeed, this theorem has already been demonstrated (or, better, is obvious) in terms of Definition 27, and it remains to establish the equivalence of Definitions 26127.

Conceptual proof. The linear subspace of $\mathbb{R}^{4,1}$ spanned by the isotropic vectors $\hat{x}, \hat{x}_{i}, \hat{x}_{j}, \hat{x}_{i j}$ is three-dimensional. Its orthogonal complement is therefore two-dimensional and lies in $\mathbb{R}_{\text {out }}^{4,1}$. Therefore, it represents a circle (an intersection of two spheres).

Computational proof. For arbitrary representatives $\tilde{x} \in \mathbb{L}^{4,1}$ of $\hat{x}$, the requirement of Definition 27 is equivalent to equation of the type (11). Since the representatives $\hat{x}=x+\mathbf{e}_{0}+|x|^{2} \mathbf{e}_{\infty}$ fixed in (14) lie in an affine hyperplane of $\mathbb{R}^{4,1}$ (their $\mathbf{e}_{0}$-component is equal to 1 ), one has an equation of the type (2) for them. Clearly, this holds if and only if $x$ is a Q-net in $\mathbb{R}^{3}$ and $|x|^{2}$ satisfies the same equation (2) as $x$ does. We show that the latter condition is equivalent to circularity. On a single planar elementary quadrilateral $\left(x, x_{i}, x_{i j}, x_{j}\right)$, the function $|x|^{2}$ satisfies eq. (2) simultaneously with $\mid x-$ $\left.c\right|^{2}=|x|^{2}-2\langle x, c\rangle+|c|^{2}$ with any $c \in \mathbb{R}^{3}$. Choose $c$ to be the center of the circle through the three points $x, x_{i}, x_{j}$, so that $|x-c|^{2}=\left|x_{i}-c\right|^{2}=\left|x_{j}-c\right|^{2}$. Then eq. (2) for $|x-c|^{2}$ turns into $\left|x_{i j}-c\right|^{2}=|x-c|^{2}$, which means that $x_{i j}$ lies on the same circle.

Two-dimensional circular nets $(m=2)$ are discrete analogs of the curvature lines parametrized surfaces, while the case $m=3$ discretizes orthogonal coordinate systems in $\mathbb{R}^{3}$. A construction of an elementary hexahedron of a circular net is based on the following geometric theorem:

Theorem 28 (Elementary hexahedron of a circular net) Given seven points $x, x_{i}$, and $x_{i j}(1 \leq i<j \leq 3)$ in $\mathbb{R}^{3}$, such that each of the three quadruples $\left(x, x_{i}, x_{j}, x_{i j}\right)$ lies on a circle $C_{i j}$, define three new circles $\tau_{i} C_{j k}$ as those passing through the triples $\left(x_{i}, x_{i j}, x_{i k}\right)$, respectively. Then these new circles intersect at one point, see Fig. 12:

$$
x_{123}=\tau_{1} C_{23} \cap \tau_{2} C_{31} \cap \tau_{3} C_{12} .
$$




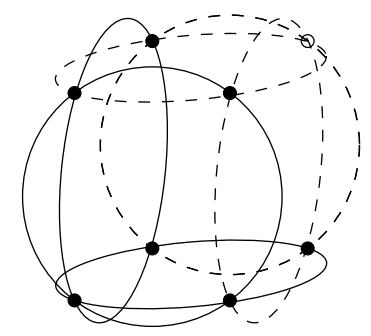

Figure 12: An elementary hexahedron of a circular net

Proof. This is a particular case of Theorem [15] applied to the quadric $\mathbb{P}\left(\mathbb{L}^{4,1}\right)$.

This theorem can be proven also by elementary geometrical considerations. If one notes that under conditions of Theorem 28 the seven points $x, x_{i}, x_{i j}$ lie on a two-dimensional sphere, and performs a stereographic projection of this sphere with the pole at $x$, one arrives at a planar picture which is nothing but the classical Miquel theorem.

\subsection{Laguerre geometry: conical nets}

Conical meshes have been introduced recently in [LPWYW].

Caution: in this section, the notation $\hat{p}$ refers to the Laguerre-geometric representatives in $\mathbb{L}^{3,1,1}$, and not to the Lie-geometric ones in $\mathbb{L}^{4,2}$. The former are obtained from the latter by omitting the (vanishing) $\mathbf{e}_{0}$-component.

As in the previous section, we assume that the principal contact element nets under consideration do not contain umbilic quadruples. The main result of this section is the following claim.

Theorem 29 (Tangent planes of principal contact element nets form conical nets) For a principal contact element net

$$
(x, P): \mathbb{Z}^{2} \rightarrow\left\{\text { contact elements of surfaces in } \mathbb{R}^{3}\right\},
$$

its tangent planes $P: \mathbb{Z}^{m} \rightarrow$ \{oriented planes in $\mathbb{R}^{3}$ \} form a conical net.

This statement refers to the notion which can be defined in two different ways. 
Definition 30 (Conical net. Euclidean model) $A$ net $P: \mathbb{Z}^{m} \rightarrow$ \{oriented planes in $\left.\mathbb{R}^{3}\right\}$ is called conical, if at any $u \in \mathbb{Z}^{m}$ and for all pairs $1 \leq i \neq j \leq m$ the four planes $P, P_{i}, P_{i j}, P_{j}$ touch a cone of revolution (in particular, intersect at the tip of the cone).

Definition 31 (Conical net. Projective model) $A$ net $P: \mathbb{Z}^{m} \rightarrow$ \{oriented planes in $\left.\mathbb{R}^{3}\right\}$ is called conical, if the corresponding $\hat{p}: \mathbb{Z}^{m} \rightarrow$ $\mathbb{P}\left(\mathbb{L}^{3,1,1}\right)$ is a $Q$-net in $\mathbb{P}\left(\mathbb{R}^{3,1,1}\right)$.

Theorem 29] is obvious in terms of Definition 31, so the real content of this theorem is the translation between the Euclidean model and the projective model, that is, establishing equivalence of Definitions 30, 31,

Proof. Representatives $\hat{p}$ in (23) form a Q-net, if and only if they satisfy eq. (2), that is, if $v: \mathbb{Z}^{m} \rightarrow \mathbb{S}^{2}$ and $d: \mathbb{Z}^{m} \rightarrow \mathbb{R}$ satisfy this equation. Equation (2) for $v$ yields that $v: \mathbb{Z}^{m} \rightarrow \mathbb{S}^{2}$ is actually a Q-net in $\mathbb{S}^{2}$, so that any quadrilateral $\left(v, v_{i}, v_{i j}, v_{j}\right)$ in $\mathbb{S}^{2}$ is planar and therefore circular. Equation (2) for $(v, d)$ yields that the (unique) intersection point of three planes $P, P_{i}, P_{j}$ lies on $P_{i j}$, as well, so that all four planes intersect in one point. Thus, we arrived at a characterization of conical nets in the sense of Definition 31 as those nets of planes for which every quadruple of planes $\left(P, P_{i}, P_{i j}, P_{j}\right)$ is concurrent and every quadrilateral $\left(v, v_{i}, v_{i j}, v_{j}\right)$ of unit normal vectors is planar. It is clear that this description is equivalent to that of Definition 30. The direction of the axis of the tangent cone coincides with the spherical center of the quadrilateral $\left(v, v_{i}, v_{i j}, v_{j}\right)$ in $\mathbb{S}^{2}$.

Thus, conical nets are Q-nets with circular Gauss maps. It is worthwhile to mention that, in order to prescribe a conical net, it is enough to prescribe a circular Gauss map $v: \mathbb{Z}^{m} \rightarrow \mathbb{S}^{2}$ and additionally the numbers $d$ (i.e., the planes $P=(v, d))$ along the coordinate axes of $\mathbb{Z}^{m}$. Indeed, these data allow one to reconstruct the conical net uniquely. This is done via a recursive procedure, whose elementary step consists in finding the fourth plane $P_{i j}$ provided three planes $P, P_{i}, P_{j}$ and the normal direction $v_{i j}$ of the fourth one are known. But this is easy: $P_{i j}$ is the plane normal to $v_{i j}$ through the unique intersection point of the three planes $P, P_{i}, P_{j}$.

\subsection{Synthesis}

In view of Theorems 25] 29, it is natural to ask whether, given a circular net $x: \mathbb{Z}^{2} \rightarrow \mathbb{R}^{3}$, or a conical net $P: \mathbb{Z}^{2} \rightarrow$ \{oriented planes in $\left.\mathbb{R}^{3}\right\}$, there exists a principal contact element net

$$
(x, P): \mathbb{Z}^{2} \rightarrow\left\{\text { contact elements of surfaces in } \mathbb{R}^{3}\right\},
$$


with the prescribed half of the data $(x$ or $P$ ). A positive answer to this question is a corollary of the following general theorem.

Theorem 32 (Extending R-congruences of spheres to curvature line parametrized surfaces) Given a discrete $R$-congruence of spheres

$$
S: \mathbb{Z}^{2} \rightarrow\left\{\text { oriented spheres in } \mathbb{R}^{3}\right\}
$$

there exists a two-parameter family of principal contact element nets

$$
(x, P): \mathbb{Z}^{2} \rightarrow\left\{\text { contact elements of surfaces in } \mathbb{R}^{3}\right\}
$$

such that $S$ belongs to the contact element $(x, P)$, i.e., $P$ is the tangent plane to $S$ at the point $x \in S$, for all $u \in \mathbb{Z}^{2}$. Such a principal contact element net is uniquely determined by prescribing a contact element $(x, P)(0,0)$ containing the sphere $S(0,0)$.

Proof. The input data is a Q-net $\hat{s}: \mathbb{Z}^{2} \rightarrow \mathbb{P}\left(\mathbb{L}^{4,2}\right)$ in the Lie quadric, and we are looking for a congruence of isotropic lines $\ell: \mathbb{Z}^{2} \rightarrow \mathcal{L}_{0}^{4,2}$ such that $\hat{s}(u) \in \ell(u)$ for all $u \in \mathbb{Z}^{2}$. The construction starts with an arbitrary isotropic line $\ell(0,0)$ through $\hat{s}(0,0)$, and hinges on the following lemma.

Lemma 33 For an isotropic line $\ell \in \mathcal{L}_{0}^{4,2}$ and a point $\hat{s}_{1} \in \mathbb{P}\left(\mathbb{L}^{4,2}\right)$ not lying on $\ell$, there is a unique isotropic line $\ell_{1}$ through $\hat{s}_{1}$ intersecting $\ell$.

Proof. Let $\hat{s}, \hat{\sigma}$ be two arbitrary points on $\ell$ (in homogeneous coordinates), so that the line $\ell$ is given by the linear combinations $\alpha \hat{s}+\beta \hat{\sigma}$. Relation $\left\langle\alpha \hat{s}+\beta \hat{\sigma}, \hat{s}_{1}\right\rangle=0$ yields

$$
\alpha: \beta=-\left\langle\hat{\sigma}, \hat{s}_{1}\right\rangle:\left\langle\hat{s}, \hat{s}_{1}\right\rangle .
$$

Thus, there exists a unique point $\hat{s}^{(1)} \in \ell$ such that $\left\langle\hat{s}^{(1)}, \hat{s}_{1}\right\rangle=0$. Now $\ell_{1}$ is the line through $\hat{s}_{1}$ and $\hat{s}^{(1)}$.

Proof of Theorem 32, continued. With the help of Lemma 33, one can construct the isotropic lines of the congruence along the coordinate axes,

$$
\ell: \mathbb{Z} \times\{0\} \rightarrow \mathcal{L}_{0}^{4,2} \text { and } \quad \ell:\{0\} \times \mathbb{Z} \rightarrow \mathcal{L}_{0}^{4,2} .
$$

Next, one has to extend the congruence $\ell$ from the coordinate axes to the whole of $\mathbb{Z}^{2}$. An elementary step of this extension consists in finding, for three given isotropic lines $\ell, \ell_{1}, \ell_{2}$ (such that $\ell$ intersects both $\ell_{1}$ and $\ell_{2}$ ) the fourth one, $\ell_{12}$, intersecting $\ell_{1}$ and $\ell_{2}$ and going through a given point $\hat{s}_{12}$. One can use for this Lemma 33. but then one has to demonstrate 
that this construction is consistent, i.e., that the lines $\ell_{12}$ obtained from the requirements of intersecting with $\ell_{1}$ and with $\ell_{2}$ coincide. We show this with the following argument. The space $V=\operatorname{span}\left(\ell, \ell_{1}, \ell_{2}\right)$ is threedimensional. The points $\hat{s}, \hat{s}_{1}, \hat{s}_{2}$ lie in $V$. By the hypothesis of the theorem, the quadrilateral $\left(\hat{s}, \hat{s}_{1}, \hat{s}_{12}, \hat{s}_{2}\right)$ is planar, therefore $\hat{s}_{12}$ lies in $V$, as well. Draw two planes in $V: \Pi_{1}=\operatorname{span}\left(\ell_{1}, \hat{s}_{12}\right)$ and $\Pi_{2}=\operatorname{span}\left(\ell_{2}, \hat{s}_{12}\right)$. Their intersection is a line $\ell_{12}$ through $\hat{s}_{12}$. It remains to prove that this line is isotropic. For this, note that $\ell_{12}$ can be alternatively described as the line through two points $\hat{s}_{1}^{(2)}=\ell_{1} \cap \ell_{12}$ and $\hat{s}_{2}^{(1)}=\ell_{2} \cap \ell_{12}$. Both these points lie in $\mathbb{P}\left(\mathbb{L}^{4,2}\right)$, since they belong to the isotropic lines $\ell_{1}$ and $\ell_{2}$, respectively. But it is easy to see that a line in $\mathbb{P}\left(\mathbb{R}^{4,2}\right)$ through two points from $\mathbb{P}\left(\mathbb{L}^{4,2}\right)$ is either isotropic, or contains no further points from $\mathbb{P}\left(\mathbb{L}^{4,2}\right)$, depending on whether these two points are polar to one another (with respect to $\mathbb{P}\left(\mathbb{L}^{4,2}\right)$ ) or not. In our case the line $\ell_{12}$ contains, by construction, one further point $\hat{s}_{12}$ from $\mathbb{P}\left(\mathbb{L}^{4,2}\right)$, therefore it has to be isotropic.

Since the representatives $\hat{x}$ in $\mathbb{P}\left(\mathbb{L}^{4,2}\right)$ of a circular net $x: \mathbb{Z}^{2} \rightarrow \mathbb{R}^{3}$ form a Q-net in $\mathbb{P}\left(\mathbb{R}^{4,2}\right)$, and the same holds for the representatives $\hat{p}$ in $\mathbb{P}\left(\mathbb{L}^{4,2}\right)$ of a conical net $P: \mathbb{Z}^{2} \rightarrow$ \{oriented planes in $\mathbb{R}^{3}$ \}, we come to the following conclusion (obtained independently by Pottmann $[\mathrm{P}]$ ).

\section{Corollary 34 (Extending circular and conical nets to principal con-} tact element nets)

i) Given a circular net $x: \mathbb{Z}^{2} \rightarrow \mathbb{R}^{3}$, there exists a two-parameter family of conical nets $P: \mathbb{Z}^{2} \rightarrow\left\{\right.$ planes in $\left.\mathbb{R}^{3}\right\}$ such that $x \in P$ for all $u \in \mathbb{Z}^{2}$, and

the contact element net

$$
(x, P): \mathbb{Z}^{2} \rightarrow\left\{\text { contact elements of surfaces in } \mathbb{R}^{3}\right\}
$$

is principal. Such a conical net is uniquely determined by prescribing a plane $P(0,0)$ through the point $x(0,0)$.

ii) Given a conical net $P: \mathbb{Z}^{2} \rightarrow\left\{\right.$ oriented planes in $\left.\mathbb{R}^{3}\right\}$, there exists a two-parameter family of circular nets $x: \mathbb{Z}^{2} \rightarrow \mathbb{R}^{3}$ such that $x \in P$ for all $u \in \mathbb{Z}^{2}$, and the contact element net

$$
(x, P): \mathbb{Z}^{2} \rightarrow\left\{\text { contact elements of surfaces in } \mathbb{R}^{3}\right\}
$$

is principal. Such a circular net is uniquely determined by prescribing a point $x(0,0)$ in the plane $P(0,0)$.

These relations can be summarized as in Fig. 13. Note that the axes of conical nets corresponding to a given circular net coincide with the Gauss map at its vertices, considered by Schief [Sch2]. 


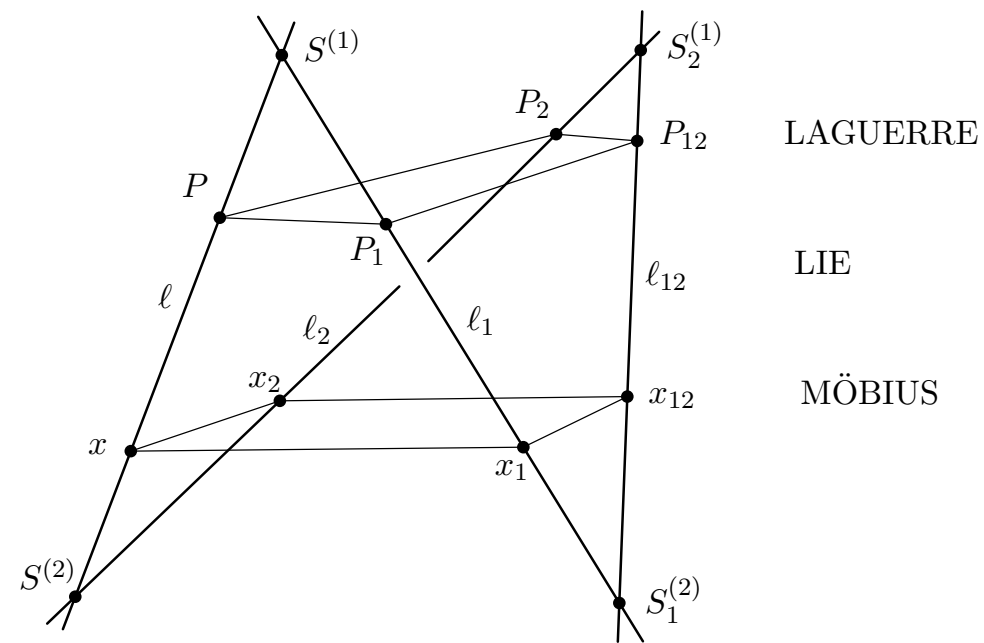

Figure 13: Elementary quadrilateral of a curvature line parametrized surface with vertices $x$ and tangent planes $P$ in the projective model. The vertices $x$ build a circular net (Möbius geometry), and lie in the planes $P$ building a conical net (Laguerre geometry). Contact elements $(x, P)$ are represented by isotropic lines $\ell$ (Lie geometry). Principal curvature spheres $S^{(i)}$ pass through pairs of neighboring points $x, x_{i}$ and are tangent to the corresponding pairs of planes $P, P_{i}$. 
Remark. In the situations of Corollary 34] i.e., when the R-congruence $S$ consists of points $x$ (and is therefore a circular net) or of planes $P$ (and is therefore a conical net), the elementary construction step of Lemma 33 allows for a very simple description from the Euclidean perspective in $\mathbb{R}^{3}$. This has been given by Pottmann $[\mathrm{P}$.

i) Given a contact element $(x, P)$ and a point $x_{1}$, find a plane $P_{1}$ through $x_{1}$ so that there exists a sphere $S^{(1)}$ tangent to both planes $P, P_{1}$ at the points $x, x_{1}$, respectively. Solution: $P_{1}$ is obtained from $P$ by the reflection in the bisecting orthogonal plane of the edge $\left[x, x_{1}\right]$. The center $c^{(1)}$ of the sphere $S^{(1)}$ is found as the intersection of the normal to $P$ at $x$ with the bisecting orthogonal plane of the edge $\left[x, x_{1}\right]$.

ii) Given a contact element $(x, P)$ and a plane $P_{1}$, find a point $x_{1}$ in $P_{1}$ so that there exists a sphere $S^{(1)}$ tangent to both planes $P, P_{1}$ at the points $x, x_{1}$, respectively. Solution: the point $x_{1}$ is obtained from $x$ by the reflection in the bisecting plane of the dihedral angle formed by $P, P_{1}$. The center $c^{(1)}$ of the sphere $S^{(1)}$ is found as the intersection of the normal to $P$ at $x$ with this bisecting plane.

\section{R-congruences of spheres}

In Sect. 4. Corollaries 21, 24, we have seen that principal curvature spheres of a principal contact element net and spheres of a Ribaucour transformation build discrete R-congruences, introduced in Definition 20. In this section we study the geometry of discrete R-congruences of spheres. Definition 20 can be re-formulated as follows: a map

$$
S: \mathbb{Z}^{2} \rightarrow\left\{\text { oriented spheres in } \mathbb{R}^{3}\right\},
$$

or the corresponding map

$$
\hat{s}: \mathbb{Z}^{2} \rightarrow \mathbb{L}^{4,2} \subset \mathbb{R}^{4,2}
$$

into the space of homogeneous coordinates, is called a discrete R-congruence of spheres, if for any $u \in \mathbb{Z}^{m}$ and for any pair $1 \leq i \neq j \leq m$ the linear subspace

$$
\Sigma=\operatorname{span}\left(\hat{s}, \hat{s}_{i}, \hat{s}_{j}, \hat{s}_{i j}\right)
$$

is three-dimensional. Thus, to any elementary square of $\mathbb{Z}^{m}$ there corresponds a three-dimensional linear subspace $\Sigma \subset \mathbb{R}^{4,2}$. 
The R-congruence of principal curvature spheres $S^{(i)}$ of the $i$-th coordinate direction is degenerate in the sense that the subspaces of its elementary quadrilaterals

$$
\Sigma=\operatorname{span}\left(\hat{s}^{(i)}, \hat{s}_{i}^{(i)}, \hat{s}_{i j}^{(i)}, \hat{s}_{j}^{(i)}\right)
$$

contain two-dimensional isotropic subspaces (corresponding to $\ell_{i}$ and $\ell_{i j}$ ). The R-congruence of spheres of a generic Ribaucour transformation is, on the contrary, non-degenerate: its $\Sigma$ 's do not contain two-dimensional isotropic subspaces, and its elementary quadrilaterals are included in planar families of spheres, introduced in the following definition.

Definition 35 (Planar family of spheres) A planar family of spheres is a set of spheres whose representatives $\hat{s} \in \mathbb{P}\left(\mathbb{L}^{4,2}\right)$ are contained in a projective plane $\mathbb{P}(\Sigma)$, where $\Sigma$ is a three-dimensional linear subspace of $\mathbb{R}^{4,2}$ such that the restriction of $\langle\cdot, \cdot\rangle$ to $\Sigma$ is non-degenerate.

Thus, a planar family of spheres is an intersection $\mathbb{P}\left(\Sigma \cap \mathbb{L}^{4,2}\right)$. Clearly, there are two possibilities:

- Signature of $\left.\langle\cdot, \cdot\rangle\right|_{\Sigma}$ is $(2,1)$, so that signature of $\left.\langle\cdot, \cdot\rangle\right|_{\Sigma^{\perp}}$ is also $(2,1)$.

- Signature of $\left.\langle\cdot, \cdot\rangle\right|_{\Sigma}$ is $(1,2)$, so that signature of $\left.\langle\cdot, \cdot\rangle\right|_{\Sigma^{\perp}}$ is $(3,0)$.

It is easy to see that a planar family is one-parametric, parametrized by a circle $\mathbb{S}^{1}$. Indeed, if $e_{1}, e_{2}, e_{3}$ is an orthogonal basis of $\Sigma$ such that $\left\langle e_{1}, e_{1}\right\rangle=$ $\left\langle e_{2}, e_{2}\right\rangle=-\left\langle e_{3}, e_{3}\right\rangle=1$ (say), then the spheres of the planar family come from the linear combinations $\hat{s}=\alpha_{1} e_{1}+\alpha_{2} e_{2}+e_{3}$ with

$$
\left\langle\alpha_{1} e_{1}+\alpha_{2} e_{2}+e_{3}, \alpha_{1} e_{1}+\alpha_{2} e_{2}+e_{3}\right\rangle=0 \quad \Leftrightarrow \quad \alpha_{1}^{2}+\alpha_{2}^{2}=1 .
$$

In the second of the cases mentioned above, the space $\Sigma^{\perp}$ only has a trivial intersection with $\mathbb{L}^{4,2}$, so that the spheres of the planar family $\mathbb{P}\left(\mathbb{L}^{4,2} \cap \Sigma\right)$ have no common touching spheres. This case has no counterpart in the smooth differential geometry. From the point of view of discrete differential geometry the first case is more significant.

Definition 36 (Cyclidic family of spheres) A planar family of spheres is called cyclidic, if the signature of $\left.\langle\cdot, \cdot\rangle\right|_{\Sigma}$ is $(2,1)$, so that the signature of $\left.\langle\cdot, \cdot\rangle\right|_{\Sigma^{\perp}}$ is also $(2,1)$.

Thus, for a cyclidic family $\mathbb{P}\left(\mathbb{L}^{4,2} \cap \Sigma\right)$ there is a dual cyclidic family $\mathbb{P}\left(\mathbb{L}^{4,2} \cap \Sigma^{\perp}\right)$ such that any sphere of the first one is in an oriented contact 
with any sphere of the second one. The family $\mathbb{P}\left(\mathbb{L}^{4,2} \cap \Sigma\right)$, as any oneparameter family of spheres, envelopes a canal surface in $\mathbb{R}^{3}$, and this surface is an envelope of the dual family $\mathbb{P}\left(\mathbb{L}^{4,2} \cap \Sigma^{\perp}\right)$, as well. Such surfaces are called Dupin cyclides. Thus, to any elementary quadrilateral of a discrete R-congruence whose spheres $\left(\hat{s}, \hat{s}_{i}, \hat{s}_{i j}, \hat{s}_{j}\right)$ span a subspace of the signature $(2,1)$ there corresponds a Dupin cyclide.

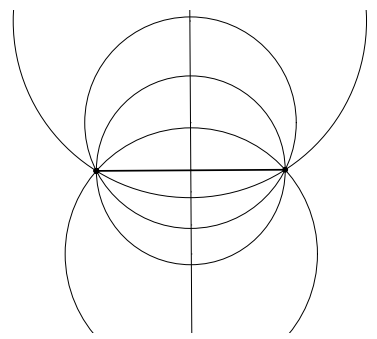

Figure 14: A cyclidic family of spheres through a circle

Examples:

- points of a circle build a planar cyclidic family of spheres (of radius zero). The dual family consists of all (oriented) spheres through this circle, with centers lying on the line through the center of the circle orthogonal to its plane, see. Fig. 14. The corresponding Dupin cyclide is the circle itself. It can be shown that any Dupin cyclide is an image of this case under a Lie sphere transformation. For a circular net, considered as a discrete R-congruence, each elementary quadrilateral carries such a structure.

- planes tangent to a cone of revolution build a planar cyclidic family of spheres, as well. The dual family consists of all (oriented) spheres tangent to the cone, with centers lying on the axis of the cone, see. Fig. 15, The corresponding Dupin cyclide is the cone itself. For a conical net, considered as a discrete R-congruence, each elementary quadrilateral carries such a structure.

Theorem 37 (Common tangent spheres of two neighboring quadrilaterals of an R-congruence) For two neighboring quadrilaterals of a discrete $R$-congruence of spheres, carrying cyclidic familes, there are generically exactly two spheres tangent to all six spheres of the congruence.

Proof. Let the quadrilaterals in question belong to the planar families generated by the subspaces $\Sigma_{1}$ and $\Sigma_{2}$ of the signature $(2,1)$. These quadrilaterals share two spheres $\hat{s}_{1}$ and $\hat{s}_{2}$, which span a linear space of the signature $(1,1)$. Each of the planar families $\Sigma_{1}$ and $\Sigma_{2}$ adds one space-like vector, 


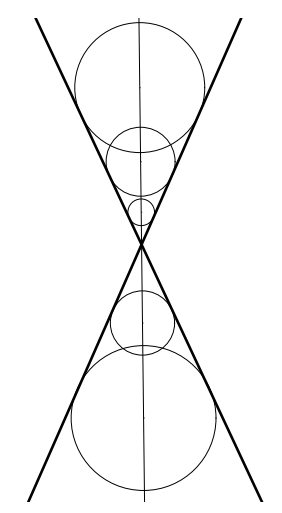

Figure 15: A cyclidic family of spheres tangent to a cone

so that the linear space $\Sigma_{1} \cup \Sigma_{2}$ spanned by all six spheres of the congruence is four-dimensional and has the signature $(3,1)$, so that its orthogonal complement $\left(\Sigma_{1} \cup \Sigma_{2}\right)^{\perp}$ is two-dimensional and has the signature $(1,1)$. Intersection of $\mathbb{L}^{4,2}$ with a two-dimensional linear subspace of the signature $(1,1)$ gives, upon projectivization, exactly two spheres: indeed, if $e_{1}, e_{2}$ form an orthogonal basis of $\left(\Sigma_{1} \cup \Sigma_{2}\right)^{\perp}$ with $\left\langle e_{1}, e_{1}\right\rangle=-\left\langle e_{2}, e_{2}\right\rangle=1$, then the spheres in this space correspond to $\alpha_{1} e_{1}+\alpha_{2} e_{2}$ with

$$
\left\langle\alpha_{1} e_{1}+\alpha_{2} e_{2}, \alpha_{1} e_{1}+\alpha_{2} e_{2}\right\rangle=0 \quad \Leftrightarrow \quad \alpha_{1}^{2}=\alpha_{2}^{2} \quad \Leftrightarrow \quad \alpha_{1}: \alpha_{2}= \pm 1 .
$$

In particular:

- For any two neighboring quadrilaterals of a circular net, there is one non-oriented sphere (hence two oriented spheres) containing both circles. Its center is the intersection point of the lines passing through the centers of the circles orthogonallly to their respective planes, see Fig. 16.

- For any two neighboring quadrilaterals of a conical net, there is a unique oriented sphere touching both cones (the second such sphere is the point at infinity). The center of this sphere is the intersection point of the axes of the cones.

The next theorem is proved in exactly the same way as Theorem 37 .

Theorem 38 (Common tangent spheres of an elementary hexahedron of an R-congruence) For an elementary hexahedron of a discrete $R$-congruence of spheres, with all faces carrying cyclidic families, there are generically exactly two spheres tangent to all eight spheres at its vertices.

It should be mentioned that these spheres, attached to elementary hexahedra, do not form a discrete R-congruence, contrary to what has been alleged by A. Doliwa as a main result of [D3]. 


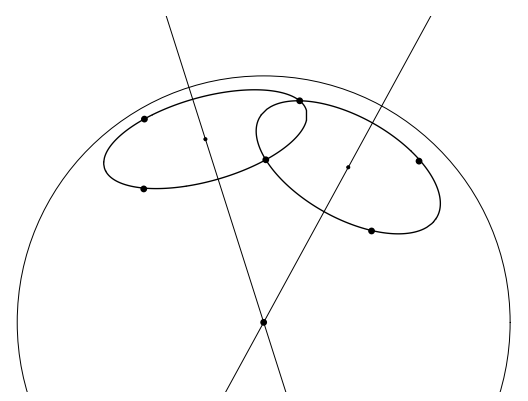

Figure 16: Normals of two neighboring quadrilaterals of a circular net intersect: both lie in the bisecting orthogonal plane of the common edge

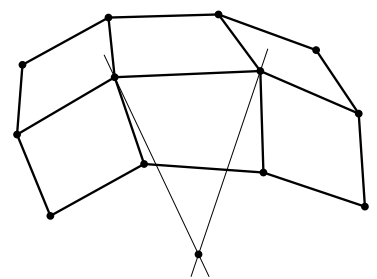

Figure 17: Normals of two neighboring quadrilaterals of a conical net intersect: two common planes of the quadrilaterals are tangent to both cones, therefore the axes of both cones lie in the bisecting plane of these two planes 
We now turn to a geometric characterization of discrete R-congruences. From eq. (44) there follows immediately that a map

$$
\left.S: \mathbb{Z}^{m} \rightarrow \text { oriented spheres in } \mathbb{R}^{3}\right\},
$$

is a discrete $\mathrm{R}$-congruence, if and only if the centers $c: \mathbb{Z}^{m} \rightarrow \mathbb{R}^{3}$ form a Q-net in $\mathbb{R}^{3}$, and the two real-valued functions,

$$
|c|^{2}-r^{2}: \mathbb{Z}^{m} \rightarrow \mathbb{R} \quad \text { and } \quad r: \mathbb{Z}^{m} \rightarrow \mathbb{R}
$$

satisfy the same equation of the type (2) as the centers $c$. By omitting the latter requirement for the signed radii $r$, one comes to a less restrictive definition than that of R-congruence. Actually, this definition belongs to Möbius geometry and refers to notations of Sect. 3.2 (with $N=3$ ).

Definition 39 (Q-congruence of spheres) A map

$$
S: \mathbb{Z}^{m} \rightarrow\left\{\text { non-oriented spheres in } \mathbb{R}^{3}\right\},
$$

is called an Q-congruence of spheres, if the corresponding map

$$
\hat{s}: \mathbb{Z}^{m} \rightarrow \mathbb{P}\left(\mathbb{R}_{\text {out }}^{4,1}\right), \quad \hat{s}=c+\mathbf{e}_{0}+\left(|c|^{2}-r^{2}\right) \mathbf{e}_{\infty},
$$

is a $Q$-net in $\mathbb{P}\left(\mathbb{R}^{4,1}\right)$.

Thus, a map (36) is a Q-congruence, if and only if the centers $c: \mathbb{Z}^{m} \rightarrow \mathbb{R}^{3}$ of the spheres $S$ form a Q-net in $\mathbb{R}^{3}$, and the function $|c|^{2}-r^{2}$ satisfies the same equation (21) as the centers $c$.

Theorem 40 (Characterization of R- among Q-congruences) Four (oriented) spheres $\left(S, S_{i}, S_{i j}, S_{j}\right)$ in $\mathbb{R}^{3}$ comprise an elementary quadrilateral of an $R$-congruence, if and only if they comprise (as non-oriented spheres) an elementary quadrilateral of a $Q$-congruence, and satisfy additionally the following condition:

(R) There exists a non-point sphere in an oriented contact with all four oriented spheres $S, S_{i}, S_{j}, S_{i j}$.

Under this condition, any sphere in an oriented contact with three spheres $S, S_{i}, S_{j}$ is in an oriented contact with the fourth one, $S_{i j}$, as well. 
Proof. Let $S_{0}$ be a sphere with the center $c_{0}$ and (finite) oriented radius $r_{0} \neq 0$ in an oriented contact with the three spheres $S, S_{i}, S_{j}$. This means that the following conditions are satisfied:

$$
\left\langle c, c_{0}\right\rangle-\frac{1}{2}\left(|c|^{2}-r^{2}\right)-\frac{1}{2}\left(\left|c_{0}\right|^{2}-r_{0}^{2}\right)-r r_{0}=0
$$

(tangency of $S, S_{0}$, cf. (10)), and two similar equation with $(c, r)$ replaced by $\left(c_{i}, r_{i}\right)$ and $\left(c_{j}, r_{j}\right)$. Now, using the fact that $c$ and $|c|^{2}-r^{2}$ satisfy one and the same equation of the type (2), we conclude that eq. (38) is fulfilled for $\left(c_{i j}, r_{i j}\right)$, if and only if $r$ satisfies the same equation (2) as $c$ and $|c|^{2}-r^{2}$ do. This proves the theorem in the case when the common tangent sphere $S_{0}$ for the three spheres $S, S_{i}, S_{j}$ has a finite radius. The case when $S_{0}$ has an infinite radius, i.e., is actually a plane, is dealt with similarly, with the help of equation

$$
\left\langle c, v_{0}\right\rangle-r-d_{0}=0
$$

which comes to replace eq. (38).

Remark. We have already seen that, generically, if three oriented spheres $S, S_{i}, S_{j}$ have a common sphere in an oriented contact, then they have a one-parameter (cyclidic) family of common touching spheres, represented by a three-dimensional linear subspace $\Sigma$ of $\mathbb{R}^{4,2}$. It is easy to see that if the projection of $\Sigma$ onto $\mathbf{e}_{\infty}^{\perp}$ is non-vanishing, then the family of spheres represented by $\Sigma^{\perp}$ contains exactly two planes. (The only exceptional case is that of a conical cyclidic family $\Sigma$, all of whose elements have vanishing $\mathbf{e}_{0}$-component and represent planes, while the family $\Sigma^{\perp}$ contains no planes.) Therefore, in all cases but the conical one, condition (R) can be replaced by the following requirement:

$\left(\mathrm{R}_{0}\right)$ The four oriented spheres $S, S_{i}, S_{j}, S_{i j}$ have a common tangent plane (actually, two common tangent planes).

It remains to give a geometric characterization of Q-congruences. This is done in the following theorem.

Theorem 41 (Three types of Q-congruences) Four (non-oriented) spheres $\left(S, S_{i}, S_{i j}, S_{j}\right)$ in $\mathbb{R}^{3}$ comprise an elementary quadrilateral of a $Q$ congruence, if and only if they satisfy one of the following three conditions:

(i) they have a common orthogonal circle, or

(ii) they intersect along a pair of points (a 0-sphere), or else

(iii) they intersect at exactly one point. 
Case (iii) can be regarded as a degenerate case of both (i) and (ii).

Conceptual proof. Caution: notations in this proof refer to the Möbiusgeometric objects which are different from the Lie-geometric objects denoted by the same symbols. The linear subspace $\Sigma$ of $\mathbb{R}^{4,1}$ spanned by the points $\hat{s}$, $\hat{s}_{i}, \hat{s}_{j}, \hat{s}_{i j}$ is three-dimensional, so that its orthogonal complement $\Sigma^{\perp}$ is twodimensional. If $\Sigma^{\perp}$ lies in $\mathbb{R}_{\text {out }}^{4,1}$, i.e., if the restriction of the Minkowski scalar product to $\Sigma^{\perp}$ is positive-definite (of signature $(2,0)$ ), then $\Sigma^{\perp}$ represents a 1-sphere (a circle) orthogonal to our four spheres, and we have the case (i). If, on the contrary, the restriction of the scalar product to $\Sigma^{\perp}$ has signature $(1,1)$, so that $\Sigma$ lies in $\mathbb{R}_{\text {out }}^{4,1}$, then $\Sigma$ represents a 0 -sphere which is the intersection of our four spheres, and we have the case (ii). Finally, if the restriction of the scalar product to $\Sigma$ is degenerate, then $\Sigma \cap \Sigma^{\perp}$ is an isotropic one-dimensional linear subspace, which represents the common point of our four spheres, and we have the case (iii).

Computational proof. The quadrilateral in $\mathbb{R}^{3}$ with the vertices at the sphere centers $c, c_{i}, c_{j}, c_{i j}$ is planar; denote its plane by $\Pi$. In the same way as in the proof of Theorem [25] we show that there is a point $C \in \Pi$ such that

$$
|c-C|^{2}-r^{2}=\left|c_{i}-C\right|^{2}-r_{i}^{2}=\left|c_{j}-C\right|^{2}-r_{j}^{2}=\left|c_{i j}-C\right|^{2}-r_{i j}^{2} .
$$

Indeed, the first two of these equations define $C$ uniquely as the intersection of two lines $\ell_{i}$ and $\ell_{j}$ in $\Pi$, where

$$
\ell_{i}=\left\{x \in \Pi:\left\langle 2 x-c_{i}-c, c_{i}-c\right\rangle=r^{2}-r_{i}^{2}\right\},
$$

and then the last equation in (40) is automatically satisfied. If the common value of all four expressions in (40) is positive (say, equal to $R^{2}$ ), then the four spheres under consideration are orthogonal to the circle in the plane $\Pi$ with the center $C$ and radius $R$, so that we have the case (i), see Fig. 18 If the common value of (40) is negative (say, equal to $-R^{2}$ ), then the pair of points on the line through $C$ orthogonal to $\Pi$, at the distance $R$ from $C$, belong to all four spheres, so that we have the case (ii). Finally, if the common value of (40) is equal to 0 , then $C$ is the intersection point of all four spheres, and we have the case (iii).

Clearly, case (i) of Q-congruences reduces to circular nets, if the radii of all spheres become infinitely small, cf. Fig. 18. Q-congruences with intersections of type (ii) are natural discrete analogs of sphere congruences parametrized along principal lines. 


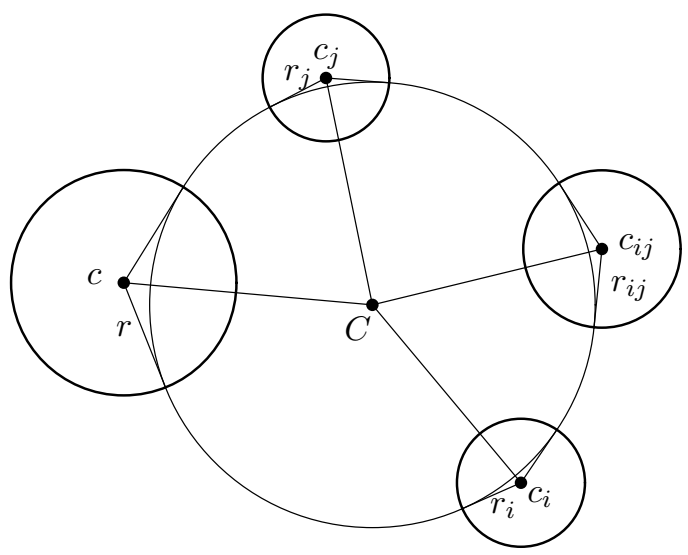

Figure 18: Elementary quadrilateral of a Q-congruence of spheres, the orthogonal circle case

Some remarks about Q-congruences of spheres are in order here. They are multidimensionally consistent, with the following reservation: given seven points $\hat{s}, \hat{s}_{i}, \hat{s}_{i j}$ in $\mathbb{P}\left(\mathbb{R}_{\text {out }}^{4,1}\right)$, the Q-property (planarity condition) uniquely defines the eighth point $\hat{s}_{123}$ in $\mathbb{P}\left(\mathbb{R}^{4,1}\right)$, which, however, might get outside of $\mathbb{P}\left(\mathbb{R}_{\text {out }}^{4,1}\right)$, and therefore might not represent a real sphere. Thus, the corresponding discrete $3 \mathrm{D}$ system is well-defined on an open subset of the space of initial data only. As long as it is defined, it can be used to produce transformations of Q-congruences, with usual permutability properties.

Note the following difference between Q-congruences and R-congruences: given three spheres $S, S_{i}, S_{j}$ of an elementary quadrilateral, one has a twoparameter family for the fourth sphere $S_{i j}$ in the case of a Q-congruence, and only a one-parameter family in the case of an R-congruence. This is a consequence of the fact that $\mathbb{R}_{\text {out }}^{4,1}$ is an open set in $\mathbb{R}^{4,1}$, while $\mathbb{L}^{4,2}$ is a hypersurface in $\mathbb{R}^{4,2}$.

\section{A Appendix: cyclographic model of Laguerre geometry}

In the cyclographic model of Laguerre geometry, the preferred space is the space of hyperspheres $\left(\mathbb{R}^{N, 1,1}\right)^{*}$, so hyperspheres $S \subset \mathbb{R}^{N}$ are modelled as 
points $\hat{s} \in \mathbb{P}\left(\left(\mathbb{R}^{N, 1,1}\right)^{*}\right)$, while hyperplanes $P \subset \mathbb{R}^{N}$ are modelled as hyperplanes $\{\xi:\langle\hat{p}, \xi\rangle=0\} \subset \mathbb{P}\left(\left(\mathbb{R}^{N, 1,1}\right)^{*}\right)$. Thus, a hyperplane $P$ is interpreted as a set of hyperspheres $S$ which are in oriented contact with $P$.

Basic features of this model:

(i) The set of oriented hyperspheres $S \subset \mathbb{R}^{N}$ is in a one-to-one correspondence with points

$$
\sigma=(c, r)
$$

of the Minkowski space $\mathbb{R}^{N, 1}$ spanned by the vectors $\mathbf{e}_{1}, \ldots, \mathbf{e}_{N}, \mathbf{e}_{N+3}$. This space has an interpretation of an affine part of $\mathbb{P}\left(\left(\mathbb{R}^{N, 1,1}\right)^{*}\right)$.

(ii) Oriented hyperplanes $P \subset \mathbb{R}^{N}$ can be modelled as hyperplanes in $\mathbb{R}^{N, 1}$ :

$$
\pi=\left\{(c, r) \in \mathbb{R}^{N, 1}:\langle(v, 1),(c, r)\rangle=\langle v, c\rangle-r=d\right\} .
$$

Thus, oriented hyperplanes $P \in \mathbb{R}^{N}$ are in a one-to-one correspondence with hyperplanes $\pi \subset \mathbb{R}^{N, 1}$ which make angle $\pi / 4$ with the subspace $\mathbb{R}^{N}=\{(x, 0)\} \subset \mathbb{R}^{N, 1}$.

(iii) An oriented hypersphere $S \subset \mathbb{R}^{N}$ is in an oriented contact with an oriented hyperplane $P \subset \mathbb{R}^{N}$, if and only if $\sigma \in \pi$.

(iv) Two oriented hyperspheres $S_{1}, S_{2} \subset \mathbb{R}^{N}$ are in an oriented contact, if and only if their representatives in the Minkowski space $\sigma_{1}, \sigma_{2} \in \mathbb{R}^{N, 1}$ differ by an isotropic vector: $\left|\sigma_{1}-\sigma_{2}\right|=0$.

In the cyclographic model, the group of Laguerre transformations admits a beautiful description:

Theorem 42 (Fundamental theorem of the Laguerre geometry) The group of Laguerre transformations is isomorphic to the group of affine transformations of $\mathbb{R}^{N, 1}: y \mapsto \lambda A y+b$, where $A \in O(N, 1), \lambda>0$, and $b \in \mathbb{R}^{N, 1}$.

\section{References}

[ABS] V.E. Adler, A.I. Bobenko, Yu.B. Suris. Classification of integrable equations on quad-graphs. The consistency approach. Commun. Math. Phys., 2003, 233, 513-543.

[AKV] A.A. Akhmetshin, I.M. Krichever, Y.S. Volvovski. Discrete analogs of the Darboux-Egoroff metrics. Proc. Steklov Inst. Math., 2 (225) (1999), 16-39. 
[Al] A.D. Alexandrov. Convex polyhedra Springer Monographs in Mathematics. Berlin: Springer, 2005, 539 p.

[ACSDLD] P. Alliez, D. Cohen-Steiner, O. Devillers, B. Levy, M. Desbrun Anisotropic Polygonal Remeshing. In: ACM SIGGRAPH Conference Proceedings 2003, 485-493

[Benz] W. Benz. Geometrische transformationen. Mannheim: BIWiss.-Verlag, 1992.

[Be] M. Berger. Geometry. Berlin etc.: Springer-Verlag, 1987.

[Bi] L. Bianchi. Lezioni di geometria differenziale. 3rd edition. Pisa: Enrico Spoerri, 1923 (Italian). iv+806, xi+832 pp.

[Bi2] L. Bianchi. Le transformazioni di Ribaucour dei sistemi $n$-pli ortogonali e il teorema generale di permutabilità. Annali di Mat., 27 (3) (1918), 183-257.

[B1] W. Blaschke. Vorlesungen über Differentialgeometrie III: Differentialgeometrie der Kreise und Kugeln. Bearbeitet von G. Thomsen. Berlin: Springer, 1929 (German). x+474 pp.

[B2] W. Blaschke. Projektive Geometrie. 3rd edition. Basel etc.: Birkhäuser, 1954. 197 pp.

[B] A.I. Bobenko. Discrete conformal maps and surfaces. - In: Symmetries and integrability of difference equations (Canterbury, 1996), CRM Proc. Lecture Notes, 255 (1999), pp. 97-108.

[BHJ] A.I. Bobenko, U. Hertrich-Jeromin. Orthogonal nets and Clifford algebras. Tôhoku Math. Publ. 20 (2001), 7-22.

[BMS] A.I. Bobenko, D. Matthes, Yu.B. Suris. Discrete and smooth orthogonal systems: $C^{\infty}$-approximation. Int. Math. Res. Not., 45 (2003), 2415-2459.

[BP1] A.I. Bobenko, U. Pinkall. Discrete surfaces with constant negative Gaussian curvature and the Hirota equation. J. Differential Geom., 43 (1996), no. 3, 527-611.

[BP2] A.I. Bobenko, U. Pinkall. Discrete isothermic surfaces. J. Reine Angew. Math., 475 (1996), 187-208. 
[BP3] A.I. Bobenko, U. Pinkall. Discretization of surfaces and integrable systems. - In: Discrete integrable geometry and physics, Eds. A.I. Bobenko and R. Seiler, Oxford: Clarendon Press, 1999, pp. $3-58$.

[BSch] A.I. Bobenko, P. Schröder. Discrete Willmore Flow. - In: Europgaphics Symposium on Geometry Processing, Eds. M. Desbrun, H. Pottmann, 2005, pp. 101-110

[BS1] A.I. Bobenko, Yu.B. Suris. Integrable systems on quad-graphs. Internat. Math. Res. Notices, 2002, Nr. 11, 573-611.

[BS2] A.I. Bobenko, Yu.B. Suris. Discrete differential geometry. Consistency as integrability. http://arxiv.org/math.DG/0504358.

[BHJ1] F. Burstall, U. Hertrich-Jeromin. Harmonic maps in unfashionable geometries. Manuscripta Math., 108 (2002), 171-189.

[BHJ2] F. Burstall, U. Hertrich-Jeromin. The Ribaucour transformation in Lie sphere geometry. http://arxiv.org/math.DG/0407244.

[C] T. Cecil. Lie sphere geometry. New York: Springer, 1992.

[CDS $]$ J. Cieslinski, A. Doliwa, P.M. Santini. The integrable discrete analogues of orthogonal coordinate systems are multidimensional circular lattices. Phys. Lett. A 235 (1997), 480-488.

[Da1] G. Darboux. Leçons sur la théorie générale des surfaces et les applications géométriques du calcul infinitésimal. T.I-IV. 3rd edition. Paris: Gauthier-Villars, 1914-1927.

[Da2] G. Darboux, Leçons sur les Systèmes Orthogonaux et les Coordonnées Curviligne, Paris: Gauthier-Villars, 1910.

[D1] A. Doliwa. Quadratic reductions of quadrilateral lattices. $J$. Geom. Phys. 30 (1999), 169-186.

[D2] A. Doliwa. Discrete asymptotic nets and $W$-congruences in Plücker line geometry. J. Geom. Phys. 39 (2001), no. 1, 9-29.

[D3] A. Doliwa. The Ribaucour congruences of spheres within Lie sphere geometry. - In: CRM Proceedings and Lecture Notes, 29 (2001), 159-166. 
[DMS] A. Doliwa, S.V. Manakov, P.M. Santini. $\bar{\partial}$ reductions of the multidimensional quadrilateral lattice: the multidimensional circular lattice. Comm. Math. Phys., 196 (1998), 1-18.

[DS] A. Doliwa, P.M. Santini. Multidimensional quadrilateral lattices are integrable. Phys. Lett. A, 233 (1997), 265-372.

[DSM] A. Doliwa, P.M. Santini, M. Mañas. Transformations of quadrilateral lattices. J. Math. Phys. 41 (2000), 944-990.

[DKG] S. Dong, S. Kircher, M. Garland. Harmonic functions for quadrilateral remeshing of arbitrary manifolds. Comp. Aided Geom. Design 22 (2005), 392-423

[Du] B. Dubrovin. Integrable systems in topological field theory. Nucl. Phys., B 379 (1992), 627-689.

[D] Ch. Dupin. Développements de géométrie. Paris: 1813.

[DN] I.A. Dynnikov, S.P. Novikov. Geometry of the triangle equation on two-manifolds. Mosc. Math. J. 3 (2003), 419-438.

[E2] L.P. Eisenhart. Transformations of surfaces. Princeton University Press, 1923. ix +379 pp.

[F1] E.V. Ferapontov. Lie sphere geometry and integrable systems. Tohoku Math. J. (2), 52 (2000), 199-233.

[F2] E.V. Ferapontov. Analog of Wilczynski's projective frame in Lie sphere geometry: Lie-applicable surfaces and commuting Schrödinger operators with magnetic fields. Internat. J. Math. 13 (2002), 959-985.

[FLPP $\quad$ D. Ferus, K. Leschke, F. Pedit, U. Pinkall. Quaternionic holomorphic geometry: Plücker formula, Dirac eigenvalue estimates and energy estimates of harmonic 2-tori. Invent. Math. 146:3 (2001), 507-593

[GT] E.I. Ganzha, S.P. Tsarev. An algebraic superposition formula and the completeness of Bäcklund transformations of $(2+1)$ dimensional integrable systems. Uspekhi Mat. Nauk 51 (1996), no. 6, 197-198 (Russian); English translation in: Russian Math. Surveys 51 (1996), 1200-1202. 
[HJ] U. Hertrich-Jeromin. Introduction to Möbius differential geometry. Cambridge University Press, 2003. xii+413 pp.

[KS] B.G. Konopelchenko, W.K. Schief. Three-dimensional integrable lattices in Euclidean spaces: conjugacy and orthogonality. $R$. Soc. Lond. Proc. Ser. A, 454 (1998), 3075-3104.

[K] I.M. Krichever. Algebraic-geometric n-orthogonal curvilinear coordinate systems and the solution of associativity equations. Funct. Anal. Appl., 31 (1997), 25-39.

[LPWYW] Y. Liu, H. Pottmann, J. Wallner, Y. Yang, and W. Wang. Geometric modeling with conical meshes and developable surfaces. ACM Trans. Graphics, 25(3) (2006), Proceedings SIGGRAPH 2006, to appear.

[MK] M. Marinov, L. Kobbelt. Direct Anisotropic Quad-Dominant Remeshing, Proc. Pacific Graphics, 2004, 207-216

$[\mathrm{MN}] \quad$ E. Musso, L. Nicolodi. On the Cauchy problem for the integrable system of Lie minimal surfaces. J. Math. Phys., 46 (2005), 113509 (15 pp.)

[N1] S.P. Novikov. Schrödinger operators on graphs and symplectic geometry. In: The Arnoldfest, Fields Inst. Commun., 24, Providence: AMS, 1999, 397-413.

[N2] S.P. Novikov. The discrete Schrödinger operator. Tr. Mat. Inst. Steklova 224 (1999) 275-290 (Russian); English translation: Proc. Steklov Inst. Math. 224 (1999) 250-265.

[ND] S.P. Novikov, I.A. Dynnikov. Discrete spectral symmetries of small-dimensional differential operators and difference operators on regular lattices and two-dimensional manifolds. Uspekhi Mat. Nauk 52 (1997), no. 5(317), 175-234 (Russian); English translation: Russian Math. Surveys 52 (1997), 1057-1116.

$[\mathrm{NM}] \quad$ A.W. Nutbourne, R.R. Martin. Differential geometry applied to curve and surface design. Vol. 1. Foundations. Ellis Horwood Limited, 1988.

[Pog] A.V. Pogorelov. Extrinsic geometry of convex surfaces, Translations of Mathematical Monographs. AMS. Providence, 1973 
[P] H. Pottmann. Generalized principal meshes and their support structures. Geometry Preprint 158, TU Wien, 2006.

[PP $] \quad$ H. Pottmann, M. Peternell. Applications of the Laguerre geometry in CAGD. Computer Aided Geom. Design, 15 (1998), $165-186$.

[RSch] C. Rogers, W.K. Schief. Bäcklund and Darboux transformations. Geometry and modern applications in soliton theory. Cambridge University Press, 2002, xviii+413 pp.

[S1] R. Sauer. Parallelogrammgitter als Modelle pseudosphärischer Flächen. Math. Z., 52 (1950), 611-622.

[S2] R. Sauer. Differenzengeometrie. Berlin etc.: Springer, 1970, 234 pp.

[Sch] W.K. Schief. On the unification of classical and novel integrable surfaces. II. Difference geometry. R. Soc. Lond. Proc. Ser. A 459 (2003), 373-391.

[Sch2] W.K. Schief. On a maximum principle for minimal surfaces and their integrable discrete counterparts. J. Geom. Phys., xxx (2005), ppp-ppp.

[T] I.A. Taimanov. Two-dimensional Dirac operator and surface theory. http://arxiv.org/math.DG/0512543.

[Wi] T.J. Willmore. Riemannian geometry., Oxford: Clarendon Press, 1993, 318 p.

[Wu] W. Wunderlich. Zur Differenzengeometrie der Flächen konstanter negativer Krümmung. Österreich. Akad. Wiss. Math.-Nat. Kl., 160 (1951), 39-77.

[Z] V.I. Zakharov, Description of the n-orthogonal curvilinear coordinate systems and Hamiltonian integrable systems of hydrodynamic type, I. Integration of the Lame equations. Duke Math. J., 94 (1998), 103-139. 